Draft Version OCTOBER 8, 2018

Preprint typeset using $\mathrm{LATE}_{\mathrm{E}} \mathrm{X}$ style emulateapj v. 01/23/15

\title{
TRIGGERING THE FORMATION OF DIRECT COLLAPSE BLACK HOLES BY THEIR CONGENERS
}

\author{
Bin Yue ${ }^{1,2}$, Andrea Ferrara ${ }^{2,3}$, Fabio PaCucci ${ }^{2,4}$, Kazuyuki Omukai ${ }^{5}$ \\ ${ }^{1}$ National Astronomical Observatories, Chinese Academy of Sciences, Beijing, 100012, China \\ ${ }^{2}$ Scuola Normale Superiore, Piazza dei Cavalieri 7, I-56126 Pisa, Italy \\ ${ }^{3}$ Kavli IPMU (WPI), Todai Institutes for Advanced Study, the University of Tokyo, Japan \\ ${ }^{4}$ Department of Physics, Yale University, P.O. Box 208101, New Haven, CT 06520, USA and \\ ${ }^{5}$ Astronomical Institute, Tohoku University, Sendai, Miyagi 980-8578, Japan \\ Draft version October 8, 2018
}

\begin{abstract}
Direct collapse black holes (DCBHs) are excellent candidates as seeds of supermassive black holes (SMBHs) observed at $z \gtrsim 6$. The formation of a DCBH requires a strong external radiation field to suppress $\mathrm{H}_{2}$ formation and cooling in a collapsing gas cloud. Such strong field is not easily achieved by first stars or normal star-forming galaxies. Here we investigate a scenario in which the previouslyformed DCBH can provide the necessary radiation field for the formation of additional ones. Using one-zone model and the simulated DCBH Spectral Energy Distributions (SEDs) filtered through absorbing gas initially having column density $N_{\mathrm{H}}$, we derive the critical field intensity, $J_{\mathrm{LW}}^{\text {crit }}$, to suppress $\mathrm{H}_{2}$ formation and cooling. For the SED model with $N_{\mathrm{H}}=1.3 \times 10^{25} \mathrm{~cm}^{-2}, 8.0 \times 10^{24} \mathrm{~cm}^{-2}$ and $5.0 \times 10^{24} \mathrm{~cm}^{-2}$, we obtain $J_{\mathrm{LW}}^{\text {crit }} \approx 22,35$ and 54 , all much smaller than the critical field intensity for normal star-forming galaxies $\left(J_{\mathrm{LW}}^{\text {crit }} \gtrsim 1000\right)$. X-ray photons from previously-formed DCBHs build up a high- $z$ X-ray background (XRB) that may boost the $J_{\mathrm{LW}}^{\text {crit }}$. However, we find that in the three SED models $J_{\mathrm{LW}}^{\text {crit }}$ only increases to $\approx 80,170$ and 390 respectively even when $\dot{\rho}_{\bullet}$ reaches the maximum value allowed by the present-day XRB level $\left(0.22,0.034,0.006 M_{\odot} \mathrm{yr}^{-1} \mathrm{Mpc}^{-3}\right)$, still much smaller than the galactic value. Although considering the XRB from first galaxies may further increase $J_{\mathrm{LW}}^{\text {crit }}$, we conclude that our investigation supports a scenario in which DCBH may be more abundant than predicted by models only including galaxies as external radiation sources.
\end{abstract}

Subject headings: dark ages, reionization, first stars - quasars: supermassive black holes - X-rays: diffuse background

\section{INTRODUCTION}

The quest for supermassive black holes (SMBHs) at $z \gtrsim 6$ has been a remarkable success (Fan et al. 2003; Jiang et al.|2007; Kurk et al. |2007; | Mortlock et al.|2011; Morganson et al. 2012; Venemans et al. 2013; Wu et al. 2015). However, the detection of such SMBHs as massive as $\sim 10^{9}-10^{10} M_{\odot}$ at such early epochs with cosmic age $\lesssim 1$ Gyr has opened a number of questions that remain essentially unanswered.

Black holes (BHs) grow from an initial seed by accreting the surrounding gas. Naive SMBH seed candidates are stellar-mass BHs formed after the death of massive (possibly Pop III) stars, with a typical mass $\sim 100 M_{\odot}$ or even smaller (Heger et al. 2003; Hosokawa et al. 2011). However, even assuming a continuous accretion at the Eddington limit, the time required to grow a $\sim 10^{9} M_{\odot}$ SMBH by $z \sim 6$ is comparable or even longer than the Hubble time at that redshift. Even worse, in the early evolutionary stages, radiative feedback reduces the accretion rate significantly. As a result, for most of the time the BH grows slowly (Alvarez et al. 2009; Jeon et al. 2012; Valiante et al. 2016). Furthermore, reionization of the intergalactic medium (IGM) may also significantly hamper the growth process (Tanaka et al. 2012).

To overcome these problems, an alternative scenario in which a SMBH grows from a seed more massive than $10^{4} M_{\odot}$ has become popular (see e.g.

yuebin@bao.ac.cn review Volonteri 2010). Such massive seeds are nicely produced by the so-called "direct collapse" process occurring in metal-free atomic-cooling halos with virial temperature $T_{\text {vir }} \gtrsim 10^{4} \mathrm{~K} \quad($ Bromm \& Loeb 2003; Koushiappas et al. 2004; Lodato \& Nataraian 2006; Begelman et al. 2006, 2008; Begelman 2010; Regan \& Haehnelt|2009; Johnson et al. |2013; Latif et al. 2013a,b, 2016; Latif \& Ferrara 2016; Latif et al. 2015, 2014; Habouzit et al. 2016; Agarwal et al. 2016b, 2012, 2013, 2014; Choi et al. 2015; Regan et al. 2014; Pacucci et al. 2015a, b; (Pacucci \& Ferrara 2015). If in these halos $\mathrm{H}_{2}$ formation and cooling is suppressed, metal-free gas can only cool via $\operatorname{Ly} \alpha$ radiation that becomes inefficient when the gas cools below $\sim 8000$ $\mathrm{K}$. Then, the gas contracts almost isothermally with $T \sim 8000 \mathrm{~K}$ and avoids fragmentation during the cloud collapse (Inavoshi et al. 2014). Eventually at the center a BH with mass $\sim 10^{4}-10^{6} M_{\odot}$ forms Ferrara et al. 2014). Thereafter such a direct collapse black hole $(\mathrm{DCBH})$ continues to accrete the gas and may merge with other BHs. A fully-fledged SMBH is then produced in time scale much shorter than the Hubble time.

The suppression of $\mathrm{H}_{2}$ formation requires either a strong Lyman-Werner (LW, $11.2<h \nu<13.6 \mathrm{eV})$ UV radiation field that directly dissociates $\mathrm{H}_{2}$, or a strong continuum radiation field from near-infrared (NIR) to UV band $(0.755<h \nu<13.6 \mathrm{eV})$ that detaches the most important $\mathrm{H}_{2}$ formation catalyst, $\mathrm{H}^{-}$. The required critical field intensity and the clustering of the dark matter halos 
determine the actual abundance of DCBHs in high- $z$ Universe (Dijkstra et al. 2008; Yue et al. 2014). The DCBH abundance has been studied by many authors and the predicted number density at $z \sim 10$ ranges from $\sim 10^{-10}$ $\mathrm{Mpc}^{-3}$ to $\sim 10^{-3} \mathrm{Mpc}^{-3}$ (e.g. Agarwal et al.|2012, 2013; Dijkstra et al. 2014; Visbal et al. 2014a; Pacucci et al. 2015a; Habouzit et al. 2016). Nevertheless such number density is much smaller than that of galaxies in the high- $z$ Universe.

Generally, in this scenario the required critical field intensity is very high. For an ideal blackbody spectrum with effective temperature $10^{4} \mathrm{~K}, J_{\nu_{13.6}}^{\text {crit }} \sim 30-300$ (Omukai 2001; Shang et al. 2010); for a realistic galaxy spectrum $J_{\nu_{13} 6}^{\text {crit }} \gtrsim 1000-10000$ (Sugimura et al. 2014; Latif et al. 2015), where $J_{\nu_{13.6}}$ is the specific intensity of the radiation field at $13.6 \mathrm{eV}$ and in units $10^{-21} \mathrm{erg}$ $\mathrm{s}^{-1} \mathrm{~cm}^{-2} \mathrm{~Hz}^{-1} \mathrm{sr}^{-1}$. Such a strong radiation field can be attained only when the radiation source is very close to the DCBH-forming gas cloud. In spite of this stringent requirement, the discovery of two high- $z$ DCBH candidates in the CANDELS/GOODS-S survey has been recently reported (Pacucci et al. 2016). Moreover, the Lyman alpha emitter (LAE) "CR7" has been observed and reported as a DCBH candidate by some authors, because its strong Ly $\alpha$ and HeII $1640 \AA$ line luminosities are hard to be provided by Pop III stars; and the two nearby Pop II galaxies are ideal sources for providing strong external radiation, although this detection is still a matter of debate (Sobral et al. 2015; Pallottini et al. 2015; Smith et al. 2016; Agarwal et al. 2016a; Smidt et al. 2016; Bowler et al. 2016; Pacucci et al. 2017).

In Yue et al. (2014) we proposed that an accreting and Compton-thick DCBH can provide a radiation field that suppresses $\mathrm{H}_{2}$ formation in a more efficient way compared with a galaxy. The advantages here are, for the Spectral Energy Distribution (SED) of a Comptonthick $\mathrm{DCBH}$, the ratio of the $\mathrm{H}^{-}$photo-detachment rate to the $\mathrm{H}_{2}$ photo-dissociation rate (this ratio is a most straightforward indicator of the $\mathrm{H}_{2}$ suppression efficiency, see, Sugimura et al. 2014; Wolcott-Green et al. 2017) is higher than for normal star-forming galaxies; and a $\mathrm{BH}$ is usually brighter than a galaxy. Moreover, there is an additional advantage: if the source also produces X-ray and ionizing photons, in general it may hamper DCBH formation Glover 2016; Regan et al. 2016; Avkutalp et al. 2014; Inavoshi \& Tanaka 2015; Johnson et al. 2014; Inayoshi \& Omukai 2011); however if the source is a Compton-thick DCBH, except a few very hard $\mathrm{X}$-ray photons (i.e. $\gtrsim 10 \mathrm{keV}$ ) during most of the accretion stage it does not emit X-rays and ionizing photons. In this case, the abundance of high- $z$ DCBHs can be higher than currently expected.

In this work, we derive the critical field intensity for the formation of a new DCBH inside a collapsing gas cloud irradiated by a nearby previously-formed DCBH by means of the one-zone model in which the whole cloud is assumed to have uniform properties. The SEDs of the DCBH are taken from numerical simulations (Pacucci et al. 2015a). We consider three SED models with initial column number densities $N_{\mathrm{H}}=1.3 \times 10^{25}$ $\mathrm{cm}^{-2}, N_{\mathrm{H}}=8.0 \times 10^{24} \mathrm{~cm}^{-2}$ and $N_{\mathrm{H}}=5.0 \times 10^{24}$ $\mathrm{cm}^{-2}$ respectively. Throughout this paper $N_{\mathrm{H}}$ refers to the column number density of the gas envelope that en- closes the accretion disc, at the time when accretion just starts. The real column density filtering the $\mathrm{BH}$ radiation is time-dependent and decreases as accretion processes.

Regarding the X-ray radiation, although during most of its accretion process the nearby DCBH only emits hard $\mathrm{X}$-ray photons that negligibly influence the $\mathrm{H}_{2}$ formation, the collective X-ray emission 1 from distant DCBHs and galaxies builds up a high- $z$ X-ray background (XRB) that ionizes the gas and may promote the $\mathrm{H}_{2}$ formation. We also investigate the impact of such high- $z \mathrm{XRB}$ on the $\mathrm{DCBH}$ formation.

An external radiation field is not the only factor that can suppress the $\mathrm{H}_{2}$ formation and lead to the formation of a DCBH. Inayoshi \& Omukai (2012) pointed out that in a protogalaxy the cold and dense accretion inflows collide with each other near the center, the shocked gas then forms a hot and dense core, where $\mathrm{H}_{2}$ formation would be suppressed due to collisional dissociation. However, Visbal et al. (2014b) argued that the density required for this scenario to occur is unable to be achieved in a realistic halo. Inayoshi et al. (2015) however proposed that the high density could be achieved by collision between two protogalaxies with a high relative velocity. On the other hand, Maver et al. (2010) proposed that when two halos merge, a massive and unstable disc could be produced by merger-driven inflows. A central core forms by accretion from this disc with a high rate $\left(\gtrsim 10^{4} \dot{M}_{\odot} \mathrm{yr}^{-1}\right.$ ), no matter whether the gas is pristine or enriched. Finally a DCBH forms in the core. This was questioned by Ferrara et al. (2013) who claimed that, if a more realistic equation-of-state is adopted, then the disc may cool quickly and the accretion rate drops, leading to a black hole with final mass $\lesssim 100 M_{\odot}$. However, a more advanced model has already been investigated by Maver et al. (2015) and it is found that it is still feasible to form a $\mathrm{DCBH}$ via halo merger. In this paper we only investigate the $\mathrm{DCBH}$ formation triggered by external radiation field.

The layout of this paper is as follows. In Section 2 we introduce the one-zone model and the DCBH SEDs. In Section 3 we present the results, including tests of our code for a pure blackbody spectrum and for a normal star-forming galaxy spectrum; the critical field intensities for DCBH SEDs and their variance when the XRB is considered. We give the conclusions and discussions in Section 4. In Appendix A we compare the optical depth of the DCBH-forming gas cloud to the LW radiation, to the $\mathrm{H}^{-}$detachment radiation and to the $\mathrm{H}_{2}^{+}$dissociation radiation. In Appendix $\mathrm{B}$ we present discussions on enhanced DCBH formation probability. In Appendix C we list the chemical reactions (Table 3) and heating/cooling functions (Tables 4 \& 5) included in our chemistry network. Throughout the paper, we use the Planck cosmology parameters (Planck Collaboration et al. 2016): $\Omega_{m}=0.308, \quad \Omega_{\Lambda}=0.692, \quad h=0.6781, \quad \Omega_{b}=0.0484, \quad n=$ 0.9677 and $\sigma_{8}=0.8149$. The transfer function is from Eisenstein \& Hu (1998).

\footnotetext{
1 Generally soft X-ray photons are trapped in the high column density matter and only hard X-ray photons contribute to the XRB. However, as the matter is swallowed by the DCBH the column density decreases, eventually soft X-ray photons can also escape from the BH (Pacucci et al. 2015a) and contribute to the $\mathrm{XRB}$ as well.
} 


\section{METHODS}

\subsection{The one-zone model}

The one-zone model developed here describes the evolution of a gas cloud that eventually collapses to form a Pop III star, a galaxy or a BH. Similar approaches have been successfully applied to a variety of problems (see e.g. Omukai 2000, 2001; Omukai et al. 2005; Sugimura et al. 2014; Visbal et al. 2014c; Inayoshi \& Tanaka 2015; Stacy \& Bromm 2007).

\subsubsection{Halo build up}

The formation of a dark matter halo is described by the "top-hat" spherical collapse scenario, in which the dark matter density $\rho_{\mathrm{d}}$ evolves as

$$
\rho_{\mathrm{d}}= \begin{cases}\frac{9 \pi^{2}}{2}\left(\frac{1+z_{\mathrm{ta}}}{1-\cos \theta}\right)^{3} \rho_{c}\left(\Omega_{m}-\Omega_{b}\right) & z>z_{\text {halt }} \\ \frac{9 \pi^{2}}{2}\left(1+z_{\mathrm{ta}}\right)^{3} \rho_{c}\left(\Omega_{m}-\Omega_{b}\right) & z \leq z_{\text {halt }},\end{cases}
$$

where $\rho_{c}$ is the Universe critical density and $z_{\text {ta }}$ is the turn-around redshift; $\theta$ is linked to redshift via

$$
\theta-\sin \theta=\pi\left(\frac{1+z_{\mathrm{ta}}}{1+z}\right)^{3 / 2},
$$

and by halting the density evolution at the redshift corresponding to $\theta_{\text {halt }}=3 \pi / 2$, we force the dark matter density not to exceed the final virialization density 2

The dark matter halo provides the gravity that causes its gas content to collapse. Ignoring the pressure, the gas density $\rho_{\mathrm{g}}$ evolves as

$$
\frac{d \rho_{\mathrm{g}}}{d t}=\frac{\rho_{\mathrm{g}}}{t_{\mathrm{ff}}},
$$

where the free-fall time scale

$$
t_{\mathrm{ff}}=\sqrt{\frac{3 \pi}{32 G\left(\rho_{\mathrm{d}}+\rho_{\mathrm{g}}\right)}} .
$$

Throughout this paper we investigate a halo with $z_{\mathrm{ta}}=$ 30.6 ; its gas eventually collapses to form a galaxy or DCBH at $z \sim 13$. In Fig. 1 we plot the evolution of the dark matter and gas density, where the initial gas density is set to be $\Omega_{b} /\left(\Omega_{m}-\Omega_{b}\right) \rho_{\mathrm{d}}\left(z_{\mathrm{ta}}\right)$. This dark matter collapse gets halted at $z \sim 20.5$ and the gas density exceeds the dark matter density at $z \sim 15$.

\subsubsection{Temperature evolution}

The temperature of the cloud is followed by solving the energy equation

$$
\frac{1}{T} \frac{d T}{d t}=(\gamma-1) \frac{1}{\rho_{\mathrm{g}}} \frac{d \rho_{\mathrm{g}}}{d t}+\frac{1}{\mu} \frac{d \mu}{d t}+(\gamma-1) \frac{\mathcal{H}-\Lambda}{n k_{\mathrm{B}} T},
$$

where $\gamma=5 / 3$ is the adiabatic index for the monatomic gas, $k_{\mathrm{B}}$ is the Boltzmann constant, $\mu$ is the molecular weight and $n=\rho_{\mathrm{g}} / \mu$ is the sum of the number density of

\footnotetext{
2 In the literature, in the top-hat spherical collapse scenario, usually the "virialization redshift" refers to the "collapse redshift", i.e. the redshift corresponding to $\theta_{\text {vir }}=2 \pi$ when the density approaches infinity. However this singularity does not happen in practice, since the gas collapse gets halted well before it.
}

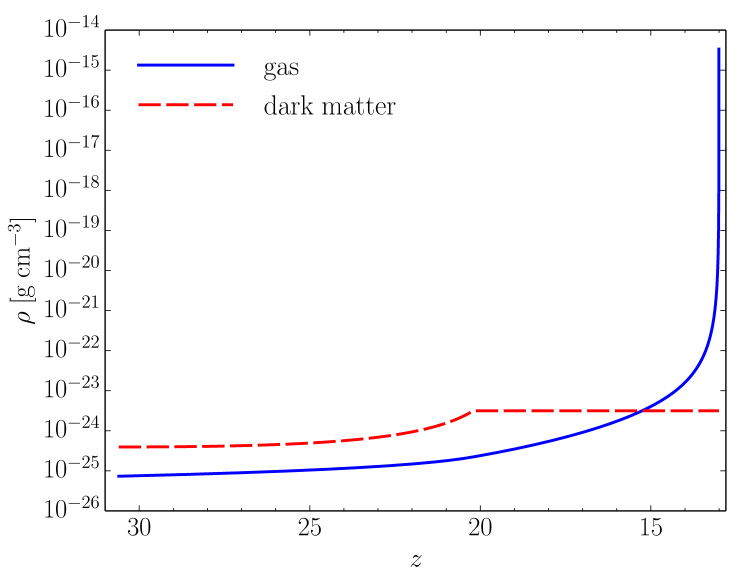

FIG. 1. - The density evolution of dark matter and collapsing gas cloud in a dark matter halo with turn-around redshift 30.6.

all the eight species; $\mathcal{H}$ and $\Lambda$ are the heating and cooling rates per unit volume, respectively.

Even in the pre-reionization epoch, X-ray photons can propagate over long distances and heat the gas with a rate

$$
\mathcal{H}_{\mathrm{X}}=f_{\mathcal{H}} \mathcal{E}
$$

where $\mathcal{E}$ is the energy (per unit time and volume) of primary electrons due to X-ray ionization, while $f_{\mathcal{H}}$ is the fraction of the primary energy deposited as thermal energy and we use the value by Valdés \& Ferrara (2008) (see Shull \& van Steenberg 1985 as well). The primary electron energy (here we ignore the photoionization of $\mathrm{He}^{+}$)

$\mathcal{E}=\int_{0.1 \mathrm{keV}} d E \frac{4 \pi J_{X}(E) e^{-\tau_{\mathrm{cl}}(E)}}{E} \sum_{i=\mathrm{H}, \mathrm{He}}\left(E-E_{i}\right) \sigma_{i}(E) n_{i}$

where $J_{X}$ is the specific intensity of the X-ray background (see Sec. 3.3), $E_{\mathrm{H}}=13.6 \mathrm{eV}$ and $E_{\mathrm{He}}=24.6 \mathrm{eV}$ are the ionization potentials for $\mathrm{H}$ and $\mathrm{He}$, respectively. The photo-electric cross-section, $\sigma_{\mathrm{H}}(E)$ and $\sigma_{\mathrm{He}}(E)$, are from Balucinska-Church \& McCammon (1992). The optical depth of the gas cloud is given as

$$
\tau_{\mathrm{cl}}(E)=\sum_{i=\mathrm{H}, \mathrm{He}} \sigma_{i}(E) n_{i} \lambda_{J} / 2
$$

by using the cloud Jeans length $\lambda_{J}$.

In addition to the X-ray heating, we also take into account the heating associated with chemical reactions listed in Table 4 in Appendix C. However, throughout this paper we are concerned with DCBH formation in the pre-reionization epoch, when the neutral IGM is opaque to UV photons, we hence ignore the ionization and heating by them.

For the cooling rate $\Lambda$, we include the radiative cooling by $\mathrm{H}_{2}, \mathrm{H}, \mathrm{He}$, and $\mathrm{He}^{+}$, and cooling associated with chemical reactions. The cooling functions for individual processes are also listed in Table 5 in Appendix C.

We stop our calculations at $n \sim 10^{9} \mathrm{~cm}^{-3}$, because our treatment of $\mathrm{H}_{2}$ cooling is only valid for number densities $\lesssim 10^{9} \mathrm{~cm}^{-3}$ when the gas is optically thin to $\mathrm{H}_{2}$ lines (e.g. Yoshida et al. 2006). However, according to some 
previous works (e.g. Omukai 2001), which investigated the subsequent evolution up to density $\gg 10^{9} \mathrm{~cm}^{-3}$, we can conclude that, as long as the $\mathrm{H}_{2}$ formation have been suppressed in a cloud before density reaches $\sim 10^{4}$ $\mathrm{cm}^{-3}, \mathrm{H}_{2}$ is collisionlly dissociated in higher density and the cloud continues to collapse only via the atomic cooling. This will result in the formation of a supermassive star at the center, which will then collapses to a $\mathrm{BH}$ (Umeda et al. 2016).

\subsection{Chemical Reactions}

We consider the evolution of eight chemical species $\mathrm{H}^{-}, \mathrm{H}, \mathrm{H}^{+}, \mathrm{H}_{2}, \mathrm{H}_{2}^{+}, \mathrm{He}, \mathrm{He}^{+}$and $e^{-}$(we ignore the $\mathrm{He}^{++}$since it plays a negligible role) by using the reaction rates listed in Table 3 in Appendix C] The reaction rates are collected from Palla et al. (1983); Omukai (2000); Glover \& Abel (2008); Shang et al. (2010) and the original references are found in them. In a cloud irradiated by an external radiation field, $\mathrm{H}_{2}$ formation is suppressed via the following three reactions: $\mathrm{H}_{2}$ photodissociation

$$
\mathrm{H}_{2}+\gamma \stackrel{k_{\mathrm{H}_{2}, \mathrm{dis}}}{\longrightarrow} 2 \mathrm{H},
$$

$\mathrm{H}^{-}$photo-detachment

$$
\mathrm{H}^{-}+\gamma \stackrel{k_{\mathrm{H}^{-}, \mathrm{det}}}{\longrightarrow} \mathrm{H}+e^{-}
$$

and $\mathrm{H}_{2}^{+}$photo-dissociation

$$
\mathrm{H}_{2}{ }^{+}+\gamma \stackrel{k_{\mathrm{H}_{2}^{+}, \mathrm{dis}}}{\longrightarrow} \mathrm{H}+\mathrm{H}^{+}
$$

respectively, where $k_{\mathrm{H}_{2} \text {,dis }}, k_{\mathrm{H}^{-} \text {,det }}$ and $k_{\mathrm{H}_{2}^{+} \text {,dis }}$ are the corresponding reaction rates (i.e., $k_{22}, k_{23}$, and $k_{24}$ in Table 3). The photo-dissociation and photo-detachement rates of $\mathrm{H}_{2}, \mathrm{H}^{-}$and $\mathrm{H}_{2}^{+}$are described below in Sec. 2.2.1. 2.2 .2 and 2.2 .3 respectively. We consider the photoionization only by the XRB (Sec. 2.2.4), and ignore all reactions that need ionizing photons other than X-rays.

\subsubsection{Photo-dissociation of $\mathrm{H}_{2}$}

Photons in the narrow LW band can dissociate the $\mathrm{H}_{2}$ through the two-step Solomon process (e.g., Stecher \& Williams 1967). The ground electronic state $\mathrm{H}_{2} X\left(v^{\prime \prime}=0, J^{\prime \prime}\right)$ are excited to the state $B\left(v^{\prime}, J^{\prime}\right)$ by absorbing the photons in the Lyman band, or to the state $C\left(v^{\prime}, J^{\prime}\right)$ by absorbing the photons in the Werner band. A fraction of these excited molecules decays to high $\left(v^{\prime \prime} \geq 14\right)$ vibrational levels and then dissociates to separate atoms. The photo-dissociation rate is then (Abel et al. 1997)

$$
k_{\mathrm{H}_{2}, \mathrm{dis}}=\sum \frac{\pi e^{2}}{m_{\mathrm{e}} c} p_{i^{\prime}} f_{i^{\prime}} \int \frac{4 \pi J(\nu)}{h \nu} \phi_{i^{\prime}}\left(\nu-\nu_{i^{\prime}}\right) d \nu,
$$

where $J(\nu)$ is the specific intensity of the external radiation field at frequency $\nu, p_{i^{\prime}}$ the probability of the transition from the ground state to $B\left(\nu^{\prime}, J^{\prime}\right)$ or $C\left(\nu^{\prime}, J^{\prime}\right)$ (Abgrall \& Roueff 1989), $f_{i^{\prime}}$ the dissociation fraction (Abgrall et al. 1992), $\nu_{i^{\prime}}$ the frequency of the transition emission line, which has normalized profile $\phi_{i^{\prime}}\left(\nu-\nu_{i^{\prime}}\right)$, and $\pi e^{2} /\left(m_{\mathrm{e}} c\right)=2.65 \times 10^{-2} \mathrm{~cm}^{2}$. We assume that the ground-state hydrogen molecules are para- $\mathrm{H}_{2}$ with
$J^{\prime \prime}=0$ (Abel et al. 1997). In case the line profile is a Dirac $\delta$ function, the above equation reduces to

$$
k_{\mathrm{H}_{2}, \mathrm{dis}} \approx \sum \frac{\pi e^{2}}{m_{\mathrm{e}} c} p_{i^{\prime}} f_{i^{\prime}} \frac{4 \pi J\left(\nu_{i^{\prime}}\right)}{h \nu_{i^{\prime}}} .
$$

The sum is performed for all possible transitions with transition energy smaller than $13.6 \mathrm{eV}$.

For a blackbody radiation field with effective temperature $10^{4} \mathrm{~K}$ ("BB") and with mean specific intensity $J_{\mathrm{LW}} \times 10^{-21} \mathrm{erg} \mathrm{s}^{-1} \mathrm{~cm}^{-2} \mathrm{~Hz}^{-1} \mathrm{sr}^{-1}$ in the LW band 3 , we have

$$
k_{\mathrm{H}_{2}, \mathrm{dis}}^{\mathrm{BB}}=7.74 \times 10^{-13} J_{\mathrm{LW}} f_{\mathrm{sh}}\left[\mathrm{s}^{-1}\right],
$$

where the self-shielding effect is included by the selfshielding parameter (Wolcott-Green et al. 2011)

$f_{\mathrm{sh}}=\frac{0.965}{\left(1+x / b_{5}\right)^{1.1}}+\frac{0.035}{(1+x)^{0.5}} \exp \left[-8.5 \times 10^{-4}(1+x)^{0.5}\right]$,

in which $x=N_{\mathrm{H}_{2}} / 5 \times 10^{14} \mathrm{~cm}^{-2}$ is the $\mathrm{H}_{2}$ column number density $N_{\mathrm{H}_{2}}$ in units $5 \times 10^{14} \mathrm{~cm}^{-2}$; and $b_{5}$ is the Doppler broadening parameter in units $10^{5} \mathrm{~cm} \mathrm{~s}^{-1}$

$$
b_{5}=\frac{\sqrt{2 k_{\mathrm{B}} T / m_{\mathrm{H}_{2}}}}{10^{5} \mathrm{~cm} \mathrm{~s}^{-1}}
$$

and $m_{\mathrm{H}_{2}}$ is the hydrogen molecule mass. We ignore the micro-turbulent velocity, therefore the self-shielding effect is possibly overestimated here (Glover \& Brand 2001). The $\mathrm{H}_{2}$ column density is given by $N_{\mathrm{H}_{2}}=$ $n_{\mathrm{H}_{2}} \lambda_{J} / 2$, where $\lambda_{J}$ is the Jeans length of the gas cloud.

For a star-forming galaxy spectrum ("GAL") with mean LW specific intensity $J_{\mathrm{LW}}$,

$$
k_{\mathrm{H}_{2}, \mathrm{dis}}^{\mathrm{GAL}}=1.23 \times 10^{-12} J_{\mathrm{LW}} f_{\mathrm{sh}}\left[\mathrm{s}^{-1}\right] .
$$

Here, we take the star-forming galaxy spectrum from STARBURST9d (Leitherer et al. 1999; Vázquez \& Leitherer 2005; Leitherer et al. 2010) and adopt the continuous star formation mode, Salpeter initial mass function (IMF) with mass range of $0.1-100$ $M_{\odot}$, metallicity of $0.02 Z_{\odot}$ and the age of $100 \mathrm{Myr}$.

For a Compton-thick DCBH, most photons in the UV to soft X-ray bands are absorbed by the thick envelope enclosing the accretion disc, then re-emitted at the energies below $13.6 \mathrm{eV}$ in the form of $\mathrm{Ly} \alpha$ emission, twophoton emission, free-free and free-bound emission, respectively. Therefore its SED has two main components: the remaining unabsorbed photons (hard X-rays and the $<13.6 \mathrm{eV}$ part of the multi-color blackbody radiation) and the re-processed photons. The relative weight of these two components depends on the column number density of the Compton-thick matter.

Using numerical simulations Pacucci et al. (2015a) have carefully investigated the radiation from DCBHs. They obtained the SEDs for different $N_{\mathrm{H}}$ values. In

3 Note that in the literature sometimes the radiation field strength is represented by different quantities. For example, in Abel et al. (1997) the specific intensity at $12.87 \mathrm{eV}$ is used, while Shang et al. (2010) uses the specific intensity at $13.6 \mathrm{eV}$. We use the mean LW specific intensity instead of the specific intensity at a given energy because our DCBH SEDs are not flat in the LW band and because of the presence of several emission lines in this band.

${ }^{4}$ http://www.stsci.edu/science/starburst99/docs/default.htm 
Fig. 2 we show the SEDs of Compton-thick DCBH with $N_{\mathrm{H}}=1.3 \times 10^{25} \mathrm{~cm}^{-2}$ ("BH1"), $8.0 \times 10^{24} \mathrm{~cm}^{-2}$ ("BH2") and $5.0 \times 10^{24} \mathrm{~cm}^{-2}$ ("BH3") respectively, at the time 10 Myr after the accretion starts. They are for the standard disk, LDP density profiles (the density profile after a $\mathrm{BH}$ has already formed at the center) with different normalizations, see Pacucci et al. (2015a) for details. We only plot the energy range relevant to the $\mathrm{H}_{2}$ chemistry, ignoring the $>13.6 \mathrm{eV}$ part. For all the three SEDs the twophoton emission is the dominant radiation from $\sim 3 \mathrm{eV}$ to $\sim 11.2 \mathrm{eV}$, below the $\sim 3 \mathrm{eV}$ the free-bound emission dominates over others. The higher the $N_{\mathrm{H}}$, the more energy is re-processed to the $<13.6 \mathrm{eV}$ part, and such $\mathrm{SED}$ is expected to suppress the $\mathrm{H}_{2}$ formation more efficiently. In case the envelope is rather thick, i.e. $N_{\mathrm{H}} \gtrsim 10^{25} \mathrm{~cm}^{-2}$, Ly $\alpha$ emission is barely seen as the corresponding photons are well-trapped in the thick envelope and, finally most of them escape from the envelope as two-photon emission.

The SED obtained in Pacucci et al. (2015a) evolves as the accretion progresses. However, for the BH mass investigated in this paper the SED is always stationary when the accretion time is smaller than $\sim 100 \mathrm{Myr}$, only the normalization changes with the increasing $\mathrm{BH}$ mass. In our work we always use the SEDs plotted in Fig. 2 as the spectrum of the external radiation field, leaving the normalization as a free parameter representing the different distances from the collapsing gas cloud to the nearby emitting DCBH.

For comparison the $\mathrm{BB}$ and GAL spectra are also shown in Fig. 2 All SEDs there have $J_{\mathrm{LW}}=1.0$. Substituting the DCBH SEDs into Eq. (13), we find all the three DCBH models have almost the same $\mathrm{H}_{2}$ photodissociation rate

$$
k_{\mathrm{H}_{2}, \mathrm{dis}}^{\mathrm{BH}}=1.30 \times 10^{-12} J_{\mathrm{LW}} f_{\mathrm{sh}}\left[\mathrm{s}^{-1}\right] .
$$

\subsubsection{Photo-detachment of $\mathrm{H}^{-}$}

The $\mathrm{H}^{-}$could be detached by photons in the wide energy range of $0.755-13.6 \mathrm{eV}$. The reaction rate is obtained through integration,

$$
k_{\mathrm{H}^{-}, \mathrm{det}}=\int_{\nu_{0.755}}^{\nu_{13.6}} \frac{4 \pi J(\nu)}{h \nu} \sigma_{\mathrm{H}^{-}, \operatorname{det}}(\nu) d \nu,
$$

where the cross-section is (Abel et al. 1997)

$$
\sigma_{\mathrm{H}^{-}, \operatorname{det}}(\nu)=7.928 \times 10^{5}\left(\nu-\nu_{0.755}\right)^{1.5} / \nu^{3}\left[\mathrm{~cm}^{2}\right] ;
$$

$\nu_{0.755}$ is the frequency (in $\mathrm{Hz}$ ) of a photon with energy $0.755 \mathrm{eV}$ and $\nu_{13.6}$ with $13.6 \mathrm{eV}$. For the BB and GAL radiation fields with mean LW specific intensity $J_{\mathrm{LW}}$, we obtain 5

$$
k_{\mathrm{H}^{-}, \mathrm{det}}^{\mathrm{BB}}=5.60 \times 10^{-8} J_{\mathrm{LW}}\left[\mathrm{s}^{-1}\right],
$$

and

$$
k_{\mathrm{H}^{-}, \mathrm{det}}^{\mathrm{GAL}}=5.43 \times 10^{-11} J_{\mathrm{LW}}\left[\mathrm{s}^{-1}\right] .
$$

5 Here the mean LW specific intensity, $J_{\mathrm{LW}}$, is only used to denote the normalization of the SED, actually all photons with energy $\gtrsim 0.755 \mathrm{eV}$ detach the $\mathrm{H}^{-}$.
For DCBH SEDs,

$$
\begin{aligned}
& k_{\mathrm{H}^{-}, \text {det }}^{\mathrm{BH}}=5.27 \times 10^{-8} J_{\mathrm{LW}}\left[\mathrm{s}^{-1}\right] \\
& k_{\mathrm{H}^{-}, \text {det }}^{\mathrm{BH}}=1.87 \times 10^{-8} J_{\mathrm{LW}}\left[\mathrm{s}^{-1}\right] \\
& k_{\mathrm{H}^{-}, \text {det }}^{\mathrm{BH}}=6.29 \times 10^{-9} J_{\mathrm{LW}}\left[\mathrm{s}^{-1}\right] .
\end{aligned}
$$

Apparently, $k_{\mathrm{H}^{-} \text {,det }}^{\mathrm{BH} 1} \approx k_{\mathrm{H}^{-} \text {,det }}^{\mathrm{BB}} \gg k_{\mathrm{H}^{-} \text {,det }}^{\mathrm{GAL}}$, and since this is the most relevant reaction suppressing the $\mathrm{H}_{2}$ formation, we therefore expect the critical specific intensity, $J_{\mathrm{LW}}^{\text {crit }}$, for a $\mathrm{BH} 1$ radiation field, to be comparable with a BB radiation field but it is much smaller than a GAL radiation field.

\subsubsection{Photo-dissociation of $\mathrm{H}_{2}^{+}$}

Here we present the photo-dissociation rate of the $\mathrm{H}_{2}^{+}$, a more careful investigation of the role played by $\mathrm{H}_{2}^{+}$ channel in $\mathrm{H}_{2}$ formation can be found in Sugimura et al. (2016), who pointed out that this channel is even more important than the $\mathrm{H}^{-}$channel in the softer radiation field. $\mathrm{H}_{2}^{+}$is dissociated by photons above $2.65 \mathrm{eV}$. The reaction has a cross-section (Shapiro \& Kang 1987) given by

$\log \left(\sigma_{\mathrm{H}_{2}^{+}}\right)=\left\{\begin{array}{r}-40.97+6.03 E_{\gamma}-0.504 E_{\gamma}^{2}+1.387 \times 10^{-2} E_{\gamma}^{3} \\ \left(2.65<E_{\gamma}<11.27\right) \\ -30.26+2.79 E_{\gamma}-0.184 E_{\gamma}^{2}+3.535 \times 10^{-3} E_{\gamma}^{3} \\ \left(11.27<E_{\gamma}<21\right)\end{array}\right.$

where $E_{\gamma}$ and the cross-section are in units $\mathrm{eV}$ and $\mathrm{cm}^{2}$ respectively. Similar to Eq. (19), we get the reaction rate for the $\mathrm{BB}$ and $\mathrm{GAL}$ radiation fields

$$
k_{\mathrm{H}_{2}^{+}, \mathrm{dis}}^{\mathrm{BB}}=4.30 \times 10^{-11} J_{\mathrm{LW}}\left[\mathrm{s}^{-1}\right] \text {, }
$$

and

$$
k_{\mathrm{H}_{2}^{+}, \mathrm{dis}}^{\mathrm{GAL}}=6.25 \times 10^{-12} J_{\mathrm{LW}}\left[\mathrm{s}^{-1}\right] .
$$

For DCBH SEDs

$$
\begin{aligned}
& k_{\mathrm{H}_{2}^{+}, \mathrm{dis}}^{\mathrm{BH} 1}=8.61 \times 10^{-10} J_{\mathrm{LW}}\left[\mathrm{s}^{-1}\right] \\
& k_{\mathrm{H}_{2}^{+}, \text {dis }}^{\mathrm{BH}}=3.63 \times 10^{-10} J_{\mathrm{LW}}\left[\mathrm{s}^{-1}\right] \\
& k_{\mathrm{H}_{2}^{+} \text {,dis }}^{\mathrm{BH} 3}=1.46 \times 10^{-10} J_{\mathrm{LW}}\left[\mathrm{s}^{-1}\right] .
\end{aligned}
$$

We have confirmed that the gas cloud is always transparent to both $\mathrm{H}^{-}$detachment radiation and $\mathrm{H}_{2}^{+}$dissociation radiation and neglect their shielding effect, see a check in Appendix $\mathrm{A}$

\subsubsection{Photo-ionization of $\mathrm{H}$ and $\mathrm{He}$ by $X$-rays}

The total ionization rate of hydrogen atoms, including photoionization and secondary ionizations, is

$$
\mathcal{I}_{\mathrm{H}}=\int d E \frac{4 \pi J(E) e^{-\tau_{\mathrm{cl}}(E)}}{E} \sigma_{\mathrm{H}}(E) n_{\mathrm{H}}+\frac{f_{\mathcal{I}, \mathrm{H}} \mathcal{E}}{E_{\mathrm{H}}},
$$

and of the helium atoms is

$$
\mathcal{I}_{\mathrm{He}}=\int d E \frac{4 \pi J(E) e^{-\tau_{\mathrm{cl}}(E)}}{E} \sigma_{\mathrm{He}}(E) n_{\mathrm{He}}+\frac{f_{\mathcal{I}, \mathrm{He}} \mathcal{E}}{E_{\mathrm{He}}},
$$

where $f_{\mathcal{I}, \mathrm{H}}$ and $f_{\mathcal{I}, \mathrm{He}}$ are fractions of primary energy deposited into hydrogen secondary ionization and helium secondary ionization respectively and are from Valdés \& Ferrara (2008). 


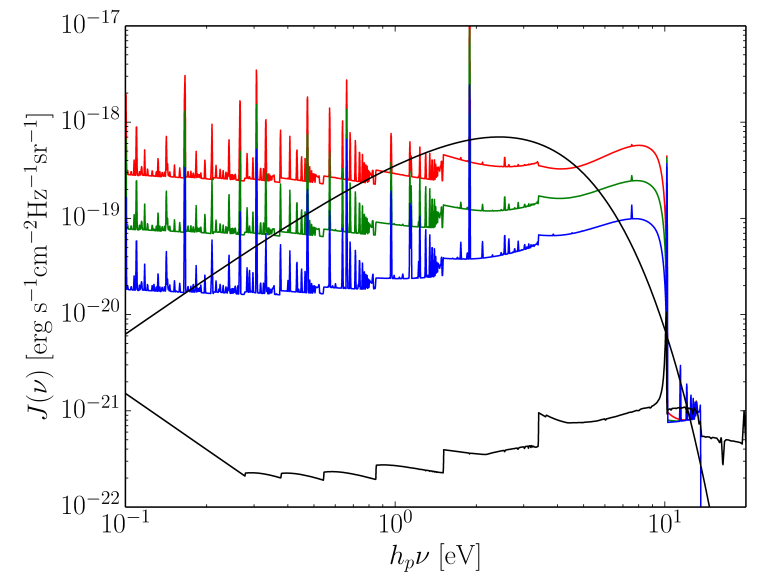

FIG. 2.- The DCBH SEDs (from top to bottom, the three curves with emission lines correspond to $\mathrm{BH} 1, \mathrm{BH} 2$ and $\mathrm{BH} 3$ respectively), compared with a blackbody spectrum with effective temperature $10^{4} \mathrm{~K}$ (smooth curves) and a star-forming galaxy spectrum (bottom curve with sawteeth).

\subsubsection{Numerical Approach}

The initial temperature is set to be the adiabatic temperature of the IGM at $z_{\text {ta }}$ (Barkana \& Loeb 2001). The initial $\mathrm{H}_{2}$ fraction, i.e. the $\mathrm{H}_{2}$ number density to $\mathrm{H}$ and $\mathrm{H}^{+}$number density ratio, is set to be $10^{-6}$ (Lepp \& Shull 1984; Hirata \& Padmanabhan 2006); the initial $\mathrm{H}^{+}$fraction and $\mathrm{He}^{+}$fraction are all set to be $10^{-4}$, and the initial electron fraction is their sum; fractions of other species are set to be zero.

We solve the differential equations of the chemistry network together with Eq. (3) and Eq. (5) starting from $z_{\text {ta }}$, to obtain the evolution of gas density, temperature and chemical abundances. To guarantee the precision we force the time-step to be the $0.1 \%$ of the minimum of all $y_{i} /\left(d y_{i} / d t\right)$, where $d y_{i} / d t$ is the derivative of the $i$ th differential equation excluding $d \mathrm{H}^{-} / d t$ and $d \mathrm{H}_{2}^{+} / d t$ (see below). The reaction rates for the intermediary species, $\mathrm{H}^{-}$and $\mathrm{H}_{2}^{+}$, are much larger than for the other species. Hence, to reduce the computational time, we always use equilibrium abundances for $\mathrm{H}^{-}$and $\mathrm{H}_{2}^{+}$obtained by iteratively solving the following simultaneous equations:

$$
\begin{aligned}
& n_{\mathrm{H}^{-}}^{\mathrm{eq}}=\left(k_{7} n_{\mathrm{H}} n_{e}+k_{12} n_{\mathrm{H}_{2}} n_{e}\right) /\left(k_{8} n_{\mathrm{H}}+k_{10} n_{\mathrm{H}_{2}^{+}}^{\mathrm{eq}}+k_{11} n_{\mathrm{H}^{+}}\right. \\
& \left.+k_{17} n_{\mathrm{e}}+k_{18} n_{\mathrm{H}}+k_{19} n_{\mathrm{H}^{+}}+k_{\mathrm{H}^{-}, \mathrm{det}}\right), \\
& n_{\mathrm{H}_{2}^{+}}^{\mathrm{eq}}=\left(k_{5} n_{\mathrm{H}} n_{\mathrm{H}^{+}}+k_{15} n_{\mathrm{H}_{2}} n_{\mathrm{H}^{+}}+k_{19} n_{\mathrm{H}^{-}}^{\mathrm{eq}} n_{\mathrm{H}^{+}}\right) /\left(k_{6} n_{\mathrm{H}}\right. \\
& \left.+k_{9} n_{\mathrm{e}}+k_{10} n_{\mathrm{H}^{-}}^{\mathrm{eq}}+k_{\mathrm{H}_{2}^{+}, \mathrm{dis}}\right) .
\end{aligned}
$$

The validity of this treatment has been proven in previous works (e.g. Abel et al. 1997; Omukai \& Nishi 1998; Bromm et al. 2002; Omukai et al. 2010).

\section{RESULTS}

\subsection{The critical intensity for the blackbody and} galactic spectra: Tests of the code

First, we run the code for a collapsing gas cloud irradiated by the $\mathrm{BB}$ and the GAL radiation fields as a test. Here we ignore the XRB.

For the $\mathrm{BB}$ radiation field with different strengths, the temperature, the $\mathrm{H}_{2}$ and $e^{-}$fractions are plotted in upper and bottom panels of Fig. 3. respectively. In the
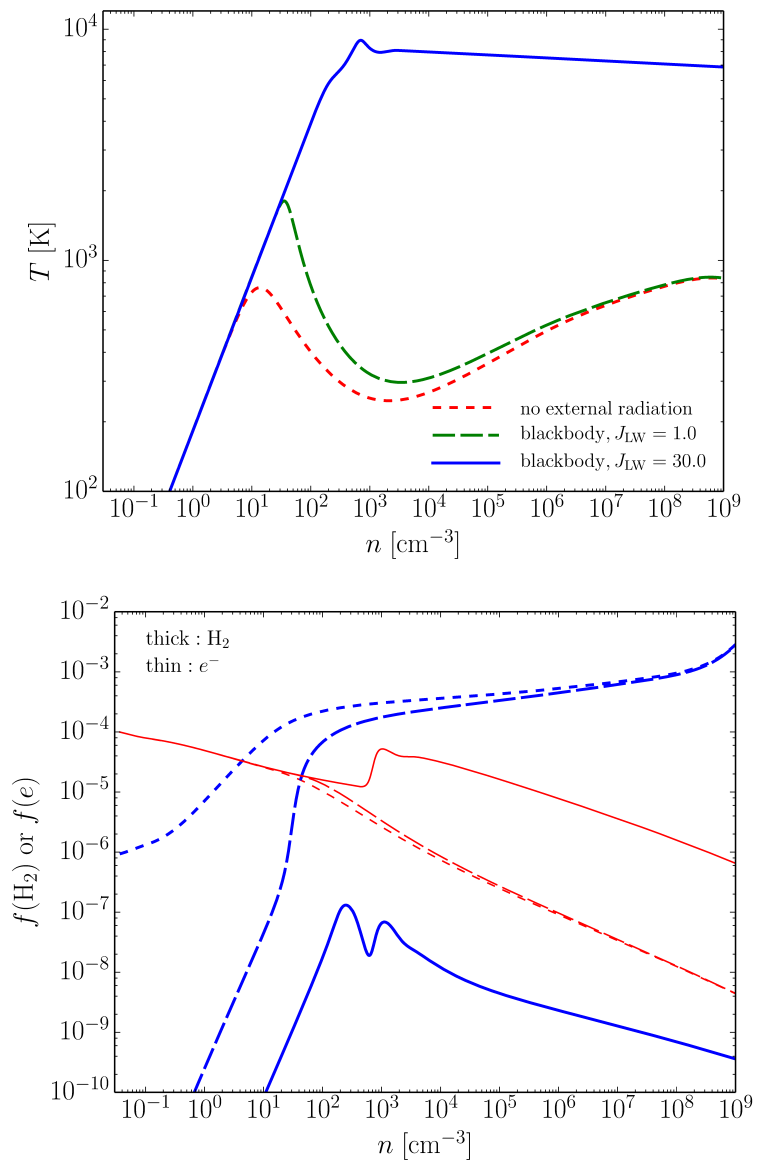

Fig. 3.- Upper panel: Temperature evolution as a function of gas density for a gas cloud irradiated by an external BB radiation field with different $J_{\mathrm{LW}}$ values. Bottom: Evolution of the $\mathrm{H}_{2}$ (thick lines) and $e^{-}$(thin lines) fractions for the BB model.

absence of an external radiation field or when the radiation field is weak (e.g. $J_{\mathrm{LW}}=1$ ), the gas cloud cools by $\mathrm{H}_{2}$ and attains a temperature $\sim 250-300 \mathrm{~K}$ at $n \sim 10^{3}$ $\mathrm{cm}^{-3}$, where the $\mathrm{H}_{2}$ rotational levels reach the local thermodynamic equilibrium. At higher densities, $\mathrm{H}_{2}$ cooling rate saturates and the temperature increases gradually, reaching $\sim 800 \mathrm{~K}$ at $n \sim 10^{9} \mathrm{~cm}^{-3}$, consistent with previous studies (Omukai 2001). On the other hand, with a more intense field, i.e., $J_{\mathrm{LW}}=30$, the $\mathrm{H}_{2}$ formation and cooling are suppressed, and up to $n \sim 10^{9} \mathrm{~cm}^{-3}$ the evolution remains quasi-isothermal at $T \gtrsim 6000 \mathrm{~K}$ set by atomic-cooling mechanism.

The blackbody spectrum with effective temperature $10^{4} \mathrm{~K}$ has $J_{\mathrm{LW}} / J_{\nu_{13.6}}=3.69$. Hence $J_{\mathrm{LW}}^{\text {crit }}=30$ is translated into $J_{\nu_{13.6}}^{\text {crit }}=8$. This is smaller than Shang et al. (2010) who found $J_{\nu_{13.6}}^{\text {crit }}=39$. Latif et al. (2014); Glover (2015) pointed out that Shang et al. (2010) ignored the dissociative tunneling effect term in Martin et al. (1996), as a result their $\mathrm{H}_{2}$ collisional dissociation rate is underestimated. Our critical value is even smaller than Glover (2015) who got $J_{\nu_{13.6}}^{\text {crit }} \approx 17$. We have checked that such discrepancy is due to the different initial setups. Using the same initial setups, and using $N_{\mathrm{H}_{2}}=n_{\mathrm{H}_{2}} \lambda_{J}$ instead of $N_{\mathrm{H}_{2}}=n_{\mathrm{H}_{2}} \lambda_{J} / 2$, we get $J_{\nu_{13.6}}^{\text {crit }}=16$, very close to Glover (2015). We further test the same initial se- 


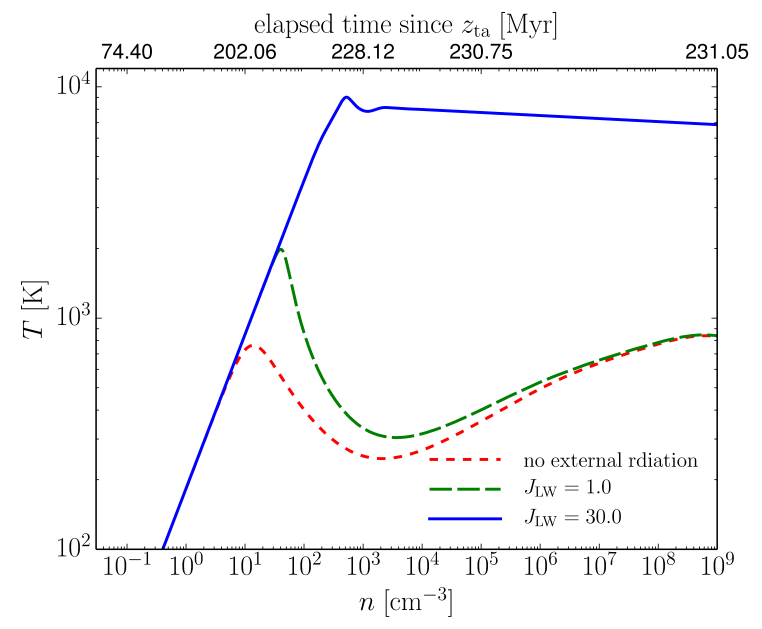

FIG. 4.- Temperature evolution for the gas cloud irradiated by the $\mathrm{BH} 1$ radiation field with different strengths. The model without external radiation field is also plotted. We mark the elapsed time since $z_{\text {ta }}$ on the upper $\mathrm{x}$-axis.

tups as Inayoshi \& Omukai (2011), and consistently get $J_{\nu_{13.6}}^{\text {crit }}=16$.

In addition to the BB radiation field, we also check the GAL field, and find that when $J_{\mathrm{LW}} \gtrsim 700$, the $\mathrm{H}_{2}$ formation and cooling is suppressed.

Regarding a Compton-thin black hole, its SED is composed of two parts with comparable bolometric luminosities (Salvaterra et al. 2005): a multi-color black body spectrum that dominates below $\lesssim 0.2 \mathrm{keV}$, and a power-law spectrum that dominates at $\gtrsim 0.2 \mathrm{keV}$. The corresponding $\mathrm{H}_{2}$ photo-dissociation rate is $\approx 1.26 \times$ $10^{-12} J_{\mathrm{LW}} f_{\mathrm{sh}}\left[\mathrm{s}^{-1}\right]$ and the $\mathrm{H}^{-}$photo-detachment rate is $\approx 6.84 \times 10^{-11} J_{\mathrm{LW}}{ }^{-1}$, and less sensitive to the black hole mass. The rates are similar to a GAL field. We therefore suspect that the critical field strength for a Comptonthin black hole is close to the GAL field. However, in addition to the photons that dissociate the $\mathrm{H}_{2}$ and detach the $\mathrm{H}^{-}$, a Compton-thin black hole simultaneously emits lots of UV and soft X-ray photons. These photons ionize and heat the collapsing gas cloud, and may either destroy it or enhance the $\mathrm{H}_{2}$ formation therein (e.g. Regan et al. 2016), resulting in a rather different critical field strength via complex mechanisms. For this reason we do not investigate such a SED in this test.

\subsection{The critical intensity by DCBHs: cases without} $X R B$

In Fig. 4. we show the temperature evolution of a collapsing gas cloud irradiated by the BH1 radiation field, for different field strengths, which corresponds to different distances to the source DCBH. For this model, we find $J_{\mathrm{LW}}^{\text {crit }} \approx 22$ and, as expected, it is similar to the $\mathrm{BB}$ radiation since $k_{\mathrm{H}^{-}, \text {det }}^{\mathrm{BB}} \approx k_{\mathrm{H}^{-}, \text {det }}^{\mathrm{BH} 1}$. For the $\mathrm{BH} 2$ and $\mathrm{BH} 3$ spectra, we find $J_{\mathrm{LW}}^{\text {crit }} \approx 35$ and $J_{\mathrm{LW}}^{\text {crit }} \approx 54$, respectively.

In the above calculations the external radiation field is turned on from the initial phase, i.e. $z_{\mathrm{ta}}=30.6$, to the final collapse redshift, i.e. $z_{\text {fin }}=13$. The time interval between these two epochs is $\approx 233$ Myr. This might raise some concern as such time span is longer than the typical DCBH accretion time, $\sim 100 \mathrm{Myr}$. We note, however, that such a long irradiation time is not really necessary.

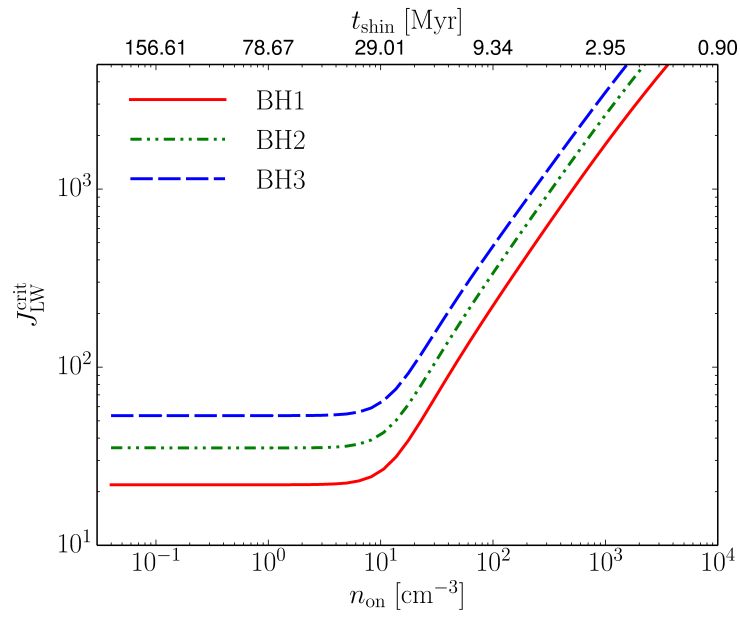

FIG. 5.- The critical intensity $J_{\mathrm{LW}}^{\mathrm{crit}}$ vs. the density at which the radiation field is switched on, $n_{\mathrm{on}}$, for different $\mathrm{DCBH}$ spectra. The shining time is marked on the upper $x$-axis.

TABLE 1

FITTING COEFFICIENTS FOR $J_{\mathrm{LW}}^{\mathrm{crit}}-n_{\text {on }}$ RELATION

\begin{tabular}{ccccc}
\hline \hline spectrum & $c_{1}$ & $c_{2}$ & $c_{3}$ & $c_{4}$ \\
\hline BH1 & 1.34 & 23.3 & 0.83 & -0.60 \\
BH2 & 1.55 & 22.3 & 0.82 & -0.60 \\
BH3 & 1.73 & 21.8 & 0.80 & -0.60 \\
\hline
\end{tabular}

For most of the time the gas density and temperature remain around the initial values. Later on, the cumulative $\mathrm{H}_{2}$ formed during this early evolutionary stages will be easily washed out by external radiation field as long as it can penetrate into the gas cloud.

We now examine the minimal requirement for the irradiation time. For this purpose, we initially set the external radiation to be zero, and switch it on at some gas density $n_{\mathrm{on}}$. We then repeat the calculations for different $n_{\mathrm{on}}$ values to obtain the dependence of $J_{\mathrm{LW}}^{\text {crit }}$ on $n_{\mathrm{on}}$. The result is presented in Fig. [5 The shining time $t_{\text {shin }}$, defined as the duration of the irradiation before the final collapse, is also indicated on the upper $x$-axis. For $n_{\text {on }} \lesssim 10 \mathrm{~cm}^{-3}, J_{\mathrm{LW}}^{\text {crit }}$ remains constant. While for higher $n_{\text {on }}$, the increasingly higher $J_{\mathrm{LW}}^{\text {crit }}$ is needed for the direct collapse. For example, with the BH1 spectrum, for $n_{\text {on }}$ as high as $100 \mathrm{~cm}^{-3}, J_{\mathrm{LW}}^{\text {crit }}$ is raised to $\approx 230$. In this case, the cloud can be irradiated only for $\sim 9 \mathrm{Myr}$ (from $n=100 \mathrm{~cm}^{-3}$ to the final collapse). If the external radiation field is switched on at $n_{\mathrm{on}}=1000 \mathrm{~cm}^{-3}$, the required intensity is even higher, $J_{\mathrm{LW}}^{\text {crit }} \approx 1800$. In this case external radiation field is only required for $\sim 3$ Myr.

The $J_{\mathrm{LW}}^{\text {crit }}-n_{\mathrm{on}}$ relation shown in Fig. 5 can be fitted by a formula

$$
\log \left(J_{\mathrm{LW}}^{\text {crit }}\right)=c_{1}+\left(1-e^{-n_{\mathrm{on}} / c_{2}}\right)\left[c_{3} \log \left(n_{\mathrm{on}}\right)+c_{4}\right]
$$

for each type of the spectrum and the coefficients $c_{1}-c_{4}$ are presented in Table 1. It may be more convenient to know the $t_{\text {shin }}$ in some cases, we therefore also fit a formula for the $J_{\mathrm{LW}}^{\text {crit }}-t_{\text {shin }}$ relation,

$$
\log \left(J_{\mathrm{LW}}^{\text {crit }}\right)=d_{1}+\left(1-e^{-d_{2} / t_{\text {shin }}}\right)\left[d_{3} \times \log \left(t_{\text {shi }}\right)+d_{4}\right],
$$


TABLE 2

FITTING COEFFICIENTS FOR $J_{\text {LW }}^{\text {crit }}-t_{\text {shin }}$ RELATION

\begin{tabular}{ccccc}
\hline \hline spectrum & $d_{1}$ & $d_{2}$ & $d_{3}$ & $d_{4}$ \\
\hline BH1 & 1.34 & 14.6 & -1.49 & 2.65 \\
BH2 & 1.55 & 14.5 & -1.45 & 2.59 \\
BH3 & 1.73 & 14.5 & -1.43 & 2.53 \\
\hline
\end{tabular}

parameters are listed in Tab. 2

\subsection{The impact of an $X R B$}

The XRB ionizes the cloud, producing more free electrons and promoting $\mathrm{H}_{2}$ formation. As such, it works as a negative feedback on $\mathrm{DCBH}$ formation and boost the critical intensity (Inavoshi \& Omukai 2011). Here, we study to what extent the critical intensity is modified by the presence of a XRB. We consider two types of the X-ray sources: (i) accreting DCBHs and (ii) first galaxies.

\subsection{1. $X$-rays from $\mathrm{DCBHs}$}

Since a DCBH is Compton-thick during most of its accretion stage, soft X-rays are absorbed in the envelope. Only hard X-rays come out from the source, which have negligible impacts on a nearby forming DCBH. However, near the end of accretion, with most of the halo gas reservoir having been swallowed by the growing $\mathrm{DCBH}$, some soft X-ray photons can leak out of the system (Pacucci et al. 2015a). Such soft X-rays from DCBHs cumulatively builds up a high- $z$ XRB.

We first derive the XRB spectrum from accreting DCBHs. Assuming that the DCBH formation rate is proportional to the formation rate of atomic-cooling halos with the virial temperature in range $10000 \mathrm{~K}<T_{\text {vir }}<$ $20000 \mathrm{~K}$, the DCBH X-ray emissivity is given by

$$
e_{X}^{\bullet}\left(E^{\prime}, z\right)=\frac{1}{4 \pi} \int_{z} L_{X}^{\bullet}\left(E^{\prime}, \Delta t^{\prime}\right) f_{\bullet} \frac{d \mathcal{N}_{\mathrm{h}}}{d z^{\prime}} d z^{\prime},
$$

where $\mathcal{N}_{\mathrm{h}}$ is the number density of atomic-cooling halos, $f$ • their fraction harboring DCBHs, $\Delta t^{\prime}$ the time interval between redshifts $z$ and $z^{\prime}$. We take the time-dependent $L_{X}^{\bullet}$ of a DCBH with initial mass $2 \times 10^{5} M_{\odot}$ from Pacucci et al. (2015a)'s numerical simulations. Since we are mainly interested in the XRB spectral shape, its amplitude is left as a free parameter. To express different XRB levels, we vary the parameter $f_{\bullet}$, which is also related to the accretion rate density by

$$
\dot{\rho}_{\bullet}(z)=\int_{z} \dot{M}_{\bullet}\left(\Delta t^{\prime}\right) f_{\bullet} \frac{d \mathcal{N}_{\mathrm{h}}}{d z^{\prime}} d z^{\prime},
$$

where $\dot{M}_{\bullet}\left(\Delta t^{\prime}\right)$ is the accretion rate onto a $\mathrm{BH}$ at a time interval $\Delta t^{\prime}$ after its birth and taken from the numerical simulations in Pacucci et al. (2015a) 6 . There are large uncertainties on the accretion rate density $\dot{\rho}_{\bullet}$, because both the occupation fraction of $\mathrm{DCBHs}$ in atomic-cooling

\footnotetext{
${ }^{6}$ Strictly speaking, BHs with different initial masses have different growth histories. Although during the growth $L_{X}^{\bullet}$ is found to be almost proportional to the BH mass in Pacucci et al. (2015a) simulations, the final XRB may depend on both the number fraction of BHs in newly-formed atomic-cooling halos and the initial mass function of BHs.
}

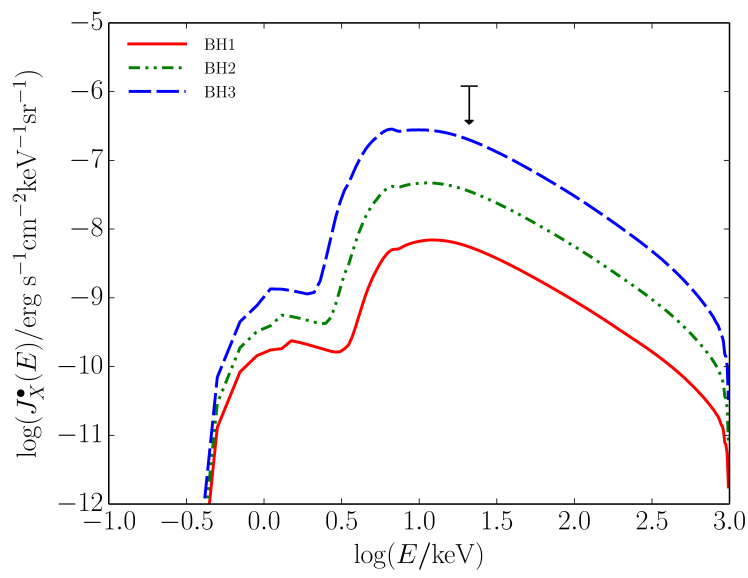

FIG. 6.- The spectral energy distributions of the XRB from DCBH populations with shielding columns $N_{\mathrm{H}}=1.3 \times 10^{25} \mathrm{~cm}^{-2}$ ("BH1"), $8.0 \times 10^{24} \mathrm{~cm}^{-2}$ ("BH2") and $5.0 \times 10^{24} \mathrm{~cm}^{-2}$ ("BH3") from the bottom to top, respectively, assuming the $\mathrm{BH}$ accretion rate density $\dot{\rho}_{\bullet}=1 \times 10^{-3} M_{\odot} \mathrm{yr}^{-1} \mathrm{Mpc}^{-3}$ at $z=13$. The arrow is the upper limit derived from the present-day observed XRB level at $1.5 \mathrm{keV}$ by Moretti et al. (2012)

halos and the $\mathrm{BH}$ mass distribution are not well-known. In the following, we therefore consider $\dot{\rho}_{\bullet}$ as a free parameter. The specific intensity of XRB at redshift $z$ is then

$$
J_{X}^{\bullet}(E, z)=(1+z)^{3} \int_{z} e_{X}^{\bullet}\left(E^{\prime}, z^{\prime}\right) e^{-\tau_{\mathrm{IGM}}\left(E^{\prime}\right)} \frac{d r_{p}}{d z^{\prime}} d z^{\prime},
$$

where $E^{\prime}=E\left(1+z^{\prime}\right) /(1+z)$, and $r_{p}$ is the proper distance. The IGM optical depth for X-ray photons is

$$
\tau_{\mathrm{IGM}}\left(E^{\prime}\right)=\int_{z}^{z^{\prime}}\left[\sum_{i=\mathrm{H}, \mathrm{He}} \bar{n}_{i}\left(z^{\prime \prime}\right) \sigma_{i}\left(E^{\prime \prime}\right)\right] \frac{d r_{p}}{d z^{\prime \prime}} d z^{\prime \prime}
$$

where $E^{\prime \prime}=E\left(1+z^{\prime \prime}\right) /(1+z)$ and $\bar{n}_{i}$ is the mean number density of species $i$ in the IGM (the trace amount metals in the IGM are ignored).

We plot the XRBs at $z=13$ for the three types of source DCBH spectra in Fig. 6 for $\dot{\rho}_{\bullet}=$ $10^{-3} M_{\odot} \mathrm{yr}^{-1} \mathrm{Mpc}^{-3}$. From the fact that the presentday intensity does not fall below the redshifted intensity from the past, i.e.,

$$
J_{X}(E, z=0) \gtrsim(1+z)^{-3} J_{X}(E(1+z), z),
$$

we can put a constraint on the XRB at high redshift from the present-day XRB from unresolved sources, $E J_{X}(1.5 \mathrm{keV}, z=0)=2 \times 10^{-13}$ erg s${ }^{-1} \mathrm{~cm}^{-2} \mathrm{deg}^{-2}$ (Moretti et al. 2012). The upper limit thus obtained for $\mathrm{XRB}$ at $z=13, J(21 \mathrm{keV}, z=13)<1.2 \times 10^{-6}$ erg $\mathrm{s}^{-1} \mathrm{~cm}^{-2} \mathrm{keV}^{-1} \mathrm{sr}^{-1}$, which is shown in Fig. 6 by a downward arrow.

Armed with the XRB spectrum above, we next investigate the impact of $\mathrm{XRB}$ on the $\mathrm{DCBH}$ formation. By repeating the temperature evolution calculations for one of the three DCBH spectra (BH1-3 with $N_{\mathrm{H}}=1.3 \times 10^{25}, 8.0 \times 10^{24}$, and $5.0 \times 10^{24} \mathrm{~cm}^{-2}$ ) with the XRB of the same $N_{\mathrm{H}}$, we can derive the critical intensity $J_{\mathrm{LW}}^{\text {crit }}$ for the DCBH formation as a function of $\dot{\rho}_{\bullet}$. The result is shown in Fig. 7 for the three DCBH spectra. For the same XRB level (i.e., $\left.\dot{\rho}_{\bullet}\right)$, the $J_{\mathrm{LW}}^{\text {crit }}$ 
increases with decreasing $N_{\mathrm{H}}$ as in the cases without the XRB. In the case of $\mathrm{BH} 1, J_{\mathrm{LW}}^{\text {crit }}$ remains $\approx 22$ be-

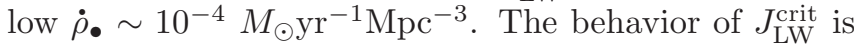
similar also for $\mathrm{BH} 2(3)$ cases: $J_{\mathrm{LW}}^{\text {crit }}$ is always $\approx 35(54)$ below $\dot{\rho}_{\bullet} \sim 10^{-5}\left(10^{-6}\right) M_{\odot} \mathrm{yr}^{-1} \mathrm{Mpc}^{-3}$.

Assuming that active DCBHs accrete at the Eddington limited rate 7 with radiative efficiency of 0.1 , we can translate the accretion rate density $\dot{\rho}_{\bullet}$ into an active $\mathrm{BH}$ mass density $\rho_{\bullet}$ :

$$
\frac{\rho_{\bullet}}{M_{\odot} \mathrm{Mpc}^{-3}} \approx 4.1 \times 10^{7} \frac{\dot{\rho}_{\bullet}}{M_{\odot} \mathrm{yr}^{-1} \mathrm{Mpc}^{-3}} .
$$

Therefore, the critical active $\mathrm{BH}$ mass densities above which the XRB gives negative feedback for the DCBH formation are about $4 \times 10^{3} M_{\odot} \mathrm{Mpc}^{-3}, 4 \times$ $10^{2} M_{\odot} \mathrm{Mpc}^{-3}$ and $4 \times 10^{1} M_{\odot} \mathrm{Mpc}^{-3}$ for the three $N_{\mathrm{H}}$ models, respectively.

With an accretion rate density $\dot{\rho}_{\bullet}$ higher than the above value, the XRB effect becomes noticeable and $J_{\mathrm{LW}}^{\text {crit }}$ increases with $\dot{\rho}_{\bullet}$. However, even with $\dot{\rho}_{\bullet}$ as high as the maximum allowed by the present-day XRB level, the DCBH can still form with feasible $J_{\mathrm{LW}}^{\text {crit }}$. For the BH1 model, the maximum allowed accretion rate density is $\approx 0.22 M_{\odot} \mathrm{yr}^{-1} \mathrm{Mpc}^{-3}$, corresponding to $\rho_{\bullet}=$ $9.0 \times 10^{6} M_{\odot} \mathrm{Mpc}^{-3}$. At this limit $J_{\mathrm{LW}}^{\text {crit }}$ only increases to $\approx 80$. Under the same hypothesis, for the $\mathrm{BH} 2(3)$ model at the maximally allowed accretion rate density, $\dot{\rho}_{\bullet}=0.034(0.006) M_{\odot} \mathrm{yr}^{-1} \mathrm{Mpc}^{-3}$, we get $J_{\mathrm{LW}}^{\text {crit }} \approx 170$ (390).

All these $J_{\mathrm{LW}}^{\text {crit }}$ values are still much smaller than those for normal star-forming galaxies, i.e. $\sim 1000-10000$ (Sugimura et al. 2014; Latif et al. 2015; Agarwal et al. 2016b). This indicates that, even with the XRB, the DCBHs are efficient radiation sources for triggering $\mathrm{DCBHs}$ in nearby halos. Moreover, an accreting $\mathrm{BH}$ is usually much brighter than a galaxy, e.g. for a DCBH with mass $10^{6} M_{\odot}$, the Eddington luminosity is $\approx 1.3 \times 10^{44} \mathrm{erg} \mathrm{s}^{-1}$, while a galaxy with our GAL spectrum and $10 M_{\odot} \mathrm{yr}^{-1}$ has bolometric luminosity $\approx 3 \times 10^{42} \mathrm{erg} \mathrm{s}^{-1}$. Hence, even if $J_{\mathrm{LW}}^{\text {crit }}$ is the same, a BH radiation can exceeds $J_{\mathrm{LW}}^{\text {crit }}$ more easily and can suppress $\mathrm{H}_{2}$ formation in a larger number of nearby metal-free halos (Yue et al. 2014). Furthermore, if a starforming galaxy is the dissociation radiation source, it also produces strong soft X-rays, which would counteract the $\mathrm{H}_{2}$ dissociation (see Sec. 3.3.2 below). However it does not happen for the surrounded DCBHs since soft X-ray photons do not leak out from the envelope during the Compton-thick stage $(\sim 100 \mathrm{Myr})$.

Finally, we evaluate the appropriate distance from a halo to bear DCBH ("hatching halo") to the radiation source ("source halo"). If the hatching halo is close enough to the source, the radiation intensity exceeds $J_{\mathrm{LW}}^{\text {crit }}$ and $\mathrm{H}_{2}$ formation is suppressed. However, if the distance among them is too small, the hatching halo could by tidally disrupted by gravity of the source halo. The tidal radius of the hatching halo is defined as

\footnotetext{
7 Note, however, that simulations by Pacucci et al. 2015a show that super-Eddington accretion may occur in highly-obscured environments, where radiation trapping is so efficient that photons are advected inward rather than being radiated away: thus, the effect of radiation pressure is dramatically reduced.
}

(Binney \& Tremaine 1987; Chon et al. 2016)

$$
r_{\mathrm{TD}}=\left(\frac{M_{\text {hat }}}{3 M_{\mathrm{sou}}}\right)^{1 / 3} d,
$$

where $M_{\text {hat }}$ and $M_{\text {sou }}$ are the masses of the hatching halo and source halos, respectively, and $d$ the distance among them. The matter of the hatching halo outside the tidal radius would be removed by the tidal force.

We conservatively require that for the hatching halo to keep its density without tidally disrupted, the tidal radius $r_{\mathrm{TD}}$ be larger than the virial radius $r_{\mathrm{vir}}$. Assuming $M_{\text {hat }} \approx M_{\text {sou }}$, we obtain $d>\sqrt[3]{3} r_{\text {vir }}=1.44 r_{\text {vir. }}$. In Fig. 7. we indicate the regions where the XRB from DCBHs is well below the present-day XRB constraints and the required $J_{\mathrm{LW}}^{\text {crit }}$ can be provided by a source DCBH of $2 \times$ $10^{5}$ or $1 \times 10^{6} M_{\odot}$ locating at the distance larger than $d>1.44 r_{\text {vir }}$, where $r_{\text {vir }}$ is evaluated for $T_{\text {vir }}=10^{4} \mathrm{~K}$ and $z=13$. Fig. 7 indicates that, for any DCBH spectrum considered, there is still a large parameter space for the DCBH formation without being tidally disrupted.

\subsubsection{X-rays from both the first galaxies and $D C B H s$}

Next, we also consider the XRB created by the first galaxies $J_{X}^{\text {gal }}$ and add it to that by DCBHs $J_{X}^{\bullet}$.

We assume the high-mass X-ray binaries (HMXBs) as the sources of XRB since they contribute most to the galactic X-ray radiation among three major contributors, i.e., HMXBs, low-mass X-ray binaries and hot interstellar medium (see e.g. Pacucci et al. 2014 for details). The Xray SED of HMXBs follows the power law with index $\alpha_{X}$, with its normalization depending on the SFR:

$$
L_{X}^{\mathrm{HMXB}}(E)=\frac{\left(1-\alpha_{X}\right) L_{\mathrm{band}}(\mathrm{SFR}, z)}{E_{2}^{\left(1-\alpha_{X}\right)}-E_{1}^{\left(1-\alpha_{X}\right)}} E^{-\alpha_{X}},
$$

where $L_{\text {band }}$ is the X-ray luminosity in the energy range considered $\left(E_{1}<E<E_{2}\right)$ and we adopt the spectral index $\alpha_{X}=1.5$ in the following. We use the fitting formula for the luminosity in $2-10 \mathrm{keV}$ (below $2 \mathrm{keV}$ the X-ray photons might be absorbed by host galaxy gas, therefore the above normalization would be not valid, see Pacucci et al. 2014) by Basu-Zych et al. (2013)

$$
\begin{aligned}
& \log \left(\frac{L_{\text {band }}}{\operatorname{erg~s}^{-1}}\right)= \\
& 0.97 \log (1+z)+0.65 \log \left(0.67 \frac{\mathrm{SFR}}{\mathrm{M}_{\odot} \mathrm{yr}^{-1}}\right)+39.80,
\end{aligned}
$$

and to model the scatter we assume a 0.5 dex variance for this relation. Although the almost linear dependence on redshift, $L_{\text {band }} \propto(1+z)^{0.97}$, is obtained from fitting to $z<4$ samples, we here extrapolate it to galaxies at higher redshifts. We have multiplied the SFR by a factor 0.67 (Madau \& Dickinson 2014) so that here the SFR corresponds to Salpeter IMF with mass limits of 0.1 and $100 M_{\odot}$, we adopted. At $z=13$ and $\mathrm{SFR}=1 M_{\odot} \mathrm{yr}^{-1}$, Eq. (40) gives $\log \left(L_{\text {band }}\right)=40.8_{-0.5}^{+0.5}$. Note that this brackets the fiducial value 40.5 adopted in Furlanetto $(2006)$.

The SFR is assumed proportional to halo mass growth rate:

$$
\operatorname{SFR}(M, z)=f_{*} \frac{\Omega_{b}}{\Omega_{m}} \frac{M}{2 \Delta t_{\mathrm{SF}}(M, z)},
$$




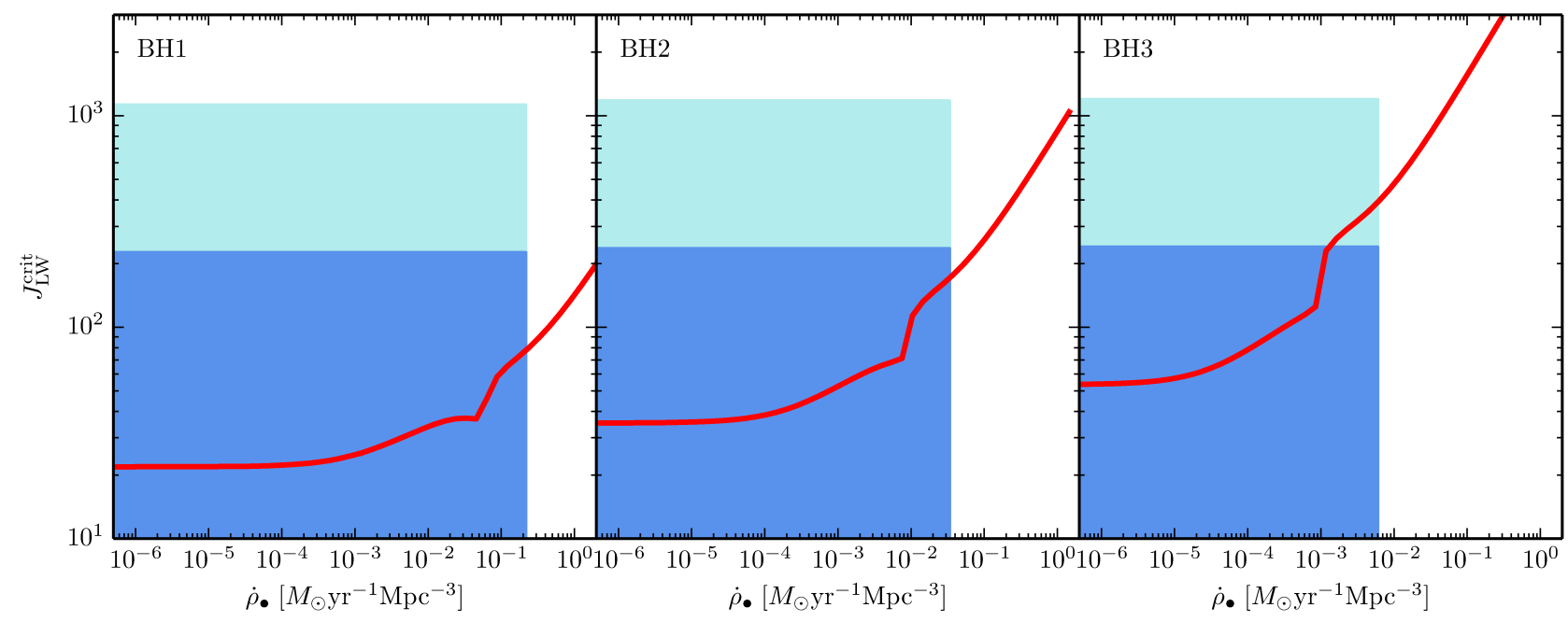

FIG. 7. $-J_{\mathrm{LW}}^{\mathrm{crit}}$ as a function of $\dot{\rho}_{\bullet}$ for three DCBH models. We fill the regions where the XRB produced by $\dot{\rho}_{\bullet}$ is below the present-day XRB level constraints, and the required $J_{\mathrm{LW}}^{\text {crit }}$ can be provided by an emitting DCBH with mass $2 \times 10^{5} M_{\odot}\left(\right.$ dark shaded) and $1 \times 10^{6} M_{\odot}$ (light \& dark shaded).

where $M$ is the halo mass, and the star formation time scale, $\Delta t_{\mathrm{SF}}$, is the time since the median formation time, which is defined as the time when the halo has collected half of its mass, to the considering redshift $z$ (Giocoli et al. 2007), and $f_{*}$ is the star formation efficiency.

The star formation efficiency $f_{*}$ is calibrated from the observed UV luminosity density $\rho_{\mathrm{UV}}$, which is derived from the UV luminosity functions of high- $z$ galaxies. By extrapolating the luminosity function in Bouwens et al. $(2015 b)$ down to the absolute UV magnitude -10 , Bouwens et al. (2015a) has obtained $\rho_{\mathrm{UV}}$ as a function of redshift and it is $\rho_{\mathrm{UV}}=10^{26.2 \pm 0.2} \mathrm{erg} \mathrm{s}^{-1} \mathrm{~Hz}^{-1} \mathrm{Mpc}^{-3}$ at $z=10$. By equating this with the sum of contributions from all halos calculated theoretically:

$$
\rho_{\mathrm{UV}}=\int_{M_{\min }} l_{\mathrm{UV}}\left(\Delta t_{\mathrm{SF}}\right) \operatorname{SFR}(M, z) \frac{d \mathcal{N}}{d M} d M,
$$

where $M_{\min }$ is the minimum halo mass that can form stars and for which we adopt the virial mass of $T_{\text {vir }}=10^{4}$ $\mathrm{K}, d \mathcal{N} / d M$ is the halo mass function (Sheth \& Tormen 1999; Sheth et al. 2001) and $l_{\mathrm{UV}}$ is the UV (at $1500 \AA$ ) luminosity per unit SFR for continuous star formation mode with Salpeter IMF $\left(0.1-100 M_{\odot}\right)$ and metallicity $Z=0.02 Z_{\odot}$, taken from STARBURST99. We then obtain $f_{*}=0.022_{-0.008}^{+0.012}$.

The XRB from first galaxies at redshift $z$ can be calculated by

$$
J_{X}^{\mathrm{gal}}(E, z)=(1+z)^{3} \int_{z} e_{X}^{\mathrm{gal}}\left(E^{\prime}, z^{\prime}\right) \mathrm{e}^{-\tau_{\mathrm{IGM}}\left(E^{\prime}\right)} \frac{d r_{\mathrm{p}}}{d z^{\prime}} d z^{\prime},
$$

where the X-ray emissivity

$$
e_{X}^{\mathrm{gal}}\left(E^{\prime}, z\right)=\frac{1}{4 \pi} \int_{M_{\min }} f_{\mathrm{esc}, \mathrm{X}}\left(E^{\prime}\right) L_{\mathrm{X}}^{\mathrm{HMXB}}\left(E^{\prime}\right) \frac{d \mathcal{N}}{d M} d M .
$$

The X-ray escape fraction $f_{\text {esc, } \mathrm{X}}$ depends on the neutral hydrogen, neutral helium and metal content of host galaxies,

$$
f_{\mathrm{esc}, \mathrm{X}}(E)=\exp \left[-\left(\sigma_{X, 1}(E) N_{\mathrm{HI}}^{\mathrm{gal}}+\sigma_{X, 2}(E) Z N_{\mathrm{HI}}^{\mathrm{gal}}\right)\right],
$$

where $N_{\mathrm{HI}}^{\mathrm{gal}}$ is the column number density of neutral hydrogen, $\sigma_{X, 1}$ and $\sigma_{X, 2}$ the synthesis photo-electric crosssection of neutral hydrogen and helium only, and of metal elements with Solar abundance, respectively Both $\sigma_{X, 1}$ and $\sigma_{X, 2}$ have been converted to those in units per hydrogen atom (Balucinska-Church \& McCammon 1992).

The neutral hydrogen column density in first galaxies is largely unknown. Lehmer et al. (2016) provide $z$ dependent expressions for the X-ray luminosity in both $0.5-2 \mathrm{keV}$ and $2-10 \mathrm{keV}$ bands. By assuming that the 2 - $10 \mathrm{keV}$ luminosity approximates the intrinsic one, while that of $0.5-2 \mathrm{keV}$ is attenuated one, and comparing these two, we derive $N_{\mathrm{HI}}^{\text {gal }} \sim 3 \times 10^{21}-3 \times 10^{22} \mathrm{~cm}^{-2}$ for SFR $\sim 10^{-3}-50 M_{\odot} \mathrm{yr}^{-1}$ at $z=13$. Although such value should be only considered as an educated guess, we nevertheless assume a fiducial value $\log N_{\mathrm{HI}}^{\text {gal }}=22$ with \pm 0.5 dex scatter, and use it for all $z \geq 13$.

In Fig. 8, we show the XRB from first galaxies at $z=13$ with the variance. They contribute $\sim 0.3 \%-6 \%$ to the unresolved present-day XRB at $1.5 \mathrm{keV}$. The total $\mathrm{XRB}$ is the sum of $J_{X}^{\text {gal }}$ and the DCBH contribution $J_{X}^{\bullet}$. Recall that the latter is a function of $\dot{\rho}_{\bullet}$. For this XRB, we re-calculate $J_{\mathrm{LW}}^{\mathrm{crit}}$ for each $\dot{\rho}_{\bullet}$. The results are plotted in Fig. 9. Comparing with the values with DCBH $\mathrm{XRB}$ only, the $J_{\mathrm{LW}}^{\mathrm{crit}}$ is boosted by about factors of 2 20. This indicates that the XRB from first galaxies may have significant negative influence on the $\mathrm{DCBH}$ formation although DCBH formation is still feasible.

\subsection{The number density of $\mathrm{DCBHs}$}

The formation rate of DCBHs is

$$
\frac{d n_{\mathrm{DCBH}}}{d z} \sim \frac{d n_{\mathrm{cool}}}{d z} P_{\mathrm{DCBH}},
$$




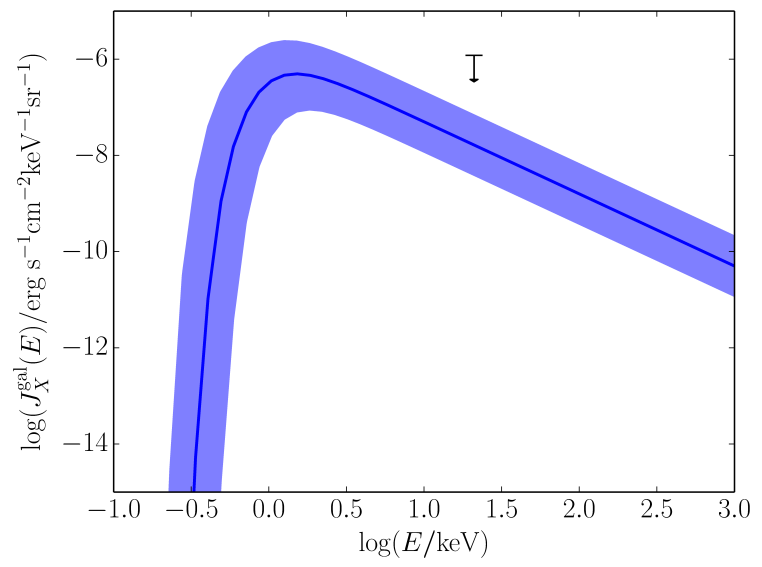

FIG. 8. - The $z=13 \mathrm{XRB}$ from first galaxies. Fiducial model with $f_{*}=0.022, \log N_{\mathrm{HI}}^{\text {gal }}=22$ and $\log L_{\text {band }}=0.93 \log (1+z)+$ $0.65 \log (0.67 \times \mathrm{SFR})+39.80$ is plotted by solid line. The variance corresponding to $f_{*}=0.022_{-0.008}^{+0.012}, \log N_{\mathrm{HI}}=22_{-0.5}^{+0.5}$ and $\log L_{\text {band }}=0.93 \log (1+z)+0.65 \log (0.67 \times \mathrm{SFR})+39.80 \pm 0.5$ is shown with a shaded region.

where $d n_{\text {cool }} / d z$ is the formation rate of atomic-cooling halos, $P_{\mathrm{DCBH}}$ is the probability that a newly-formed atomic-cooling halo matches the $\mathrm{DCBH}$ formation criteria. Here we simply assume it to be equal to the probability that this halo is located in a radiation field above the critical threshold. We ignore the metal enrichment and the radiative feedback (Yue et al. 2014; Johnson et al. 2014). This probability depends on the spatial distribution of the already-formed nearby galaxies and DCBHs. Atomic-cooling halos are assumed to host either starforming galaxies with SFR as in Eq. (41) (here we adopt $f_{*}=0.1$, or DCBHs. Using the two-point correlation function which describes the spatial distribution of their host halos, we calculate $P_{\mathrm{DCBH}}$ by the Monte Carlo simulations as in Dijkstra et al. (2008, 2014); Yue et al. (2014).

We solve the Eq. (46) from $z=25$, assuming that the initial number density of DCBHs at this redshift is zero. For simplicity we assume all DCBHs to have the same luminosity as a $5 \times 10^{5} M_{\odot}$ black hole.

In Fig. 10] we show the evolution of the DCBH number density for different SEDs and critical field strengths. In all models the final number density of DCBHs is fairly above the observed number density of SMBHs at $z \gtrsim 6$, $n_{\mathrm{SMBH}}(z \gtrsim 6) \sim 10^{-9} \mathrm{Mpc}^{-3}$. In some models $n_{\mathrm{DCBH}}$ is even above the $\sim \frac{M_{\mathrm{SMBH}}}{M_{\mathrm{DCBH}}} n_{\mathrm{SMBH}} \sim \frac{10^{9}}{10^{5}} 10^{-9}=10^{-5}$ $\mathrm{Mpc}^{-3}$. Therefore DCBHs can perform as the original seeds of SMBHs. However, we clarify that there are plenty of factors that can influence the $n_{\mathrm{DCBH}}$, for example the number of DCBHs initially formed by radiation from galaxies; the minimum separation between the collapsing cloud and the triggering source (we adopt 1.44 $r_{\text {vir }}$ as discussed in Sec. 3.3.1 1 ); the metal enrichment

8 If the triggering source is a galaxy, the minimum separation must be also larger than the ionized bubble around the galaxy. The ionized bubble radius is estimated by the equilibrium between the ionization rate and the recombination rate,

$$
\frac{4 \pi}{3} r_{\mathrm{B}}^{3} n_{\mathrm{H}}^{2} \alpha_{\mathrm{B}}=f_{\mathrm{esc}} \dot{Q}_{\mathrm{H}}
$$

where $n_{\mathrm{H}}$ is the mean gas density in the ionized bubble, $\alpha_{\mathrm{B}}$ is and radiative feedback (ignored here); the mass distribution of DCBHs (we assume a fixed mass $5 \times 10^{5} M_{\odot}$ ). Therefore what we present here is not considered as a prediction, instead it is only a proof-of-concept calculation to show how the DCBH number density should be estimated. A conclusive prediction must be based on models that treat the above factors more realistically.

\section{CONCLUSIONS AND DISCUSSION}

We have investigated the critical specific intensity of the $\mathrm{DCBH}$ radiation that could suppress $\mathrm{H}_{2}$ formation and cooling in a nearby collapsing gas cloud by one-zone calculations. If the radiation from the emitting $\mathrm{DCBH}$ is higher than the critical intensity, we expect that a new $\mathrm{DCBH}$ forms in the gas cloud. We used three realistic DCBH SED models from numerical simulations with initial column number density for the absorbing envelope $N_{\mathrm{H}}=1.3 \times 10^{25} \mathrm{~cm}^{-2}, N_{\mathrm{H}}=8.0 \times 10^{24} \mathrm{~cm}^{-2}$ and $N_{\mathrm{H}}=5.0 \times 10^{24} \mathrm{~cm}^{-2}$, respectively.

We have found that:

- DCBH spectra are very effective at photodetaching $\mathrm{H}^{-}$, a catalyst species for $\mathrm{H}_{2}$ formation. Depending on the obscuring gas column density, the ratio between $\mathrm{H}^{-}$photo-detachment and $\mathrm{H}_{2}$ photo-dissociation rates for the DCBH with $N_{\mathrm{H}}=1.3 \times 10^{25} \mathrm{~cm}^{-2}$ is $k_{\mathrm{H}^{-} \text {,det }}^{\mathrm{BH} 1} / k_{\mathrm{H}_{2} \text {,dis }}^{\mathrm{BH} 1}=4.1 \times 10^{4}$, i.e. comparable to that of a blackbody spectrum with effective temperature $10^{4} \mathrm{~K}$ which is $k_{\mathrm{H}^{-} \text {,det }}^{\mathrm{BB}} / k_{\mathrm{H}_{2} \text {,dis }}^{\mathrm{BB}}=7.2 \times 10^{4}$. For the other two DCBH SEDs the $k_{\mathrm{H}^{-} \text {,det }}^{\mathrm{BH} 2} / k_{\mathrm{H}_{2} \text {,dis }}^{\mathrm{BH} 2}=1.4 \times 10^{4}$ and $k_{\mathrm{H}^{-} \text {,det }}^{\mathrm{BH}} / k_{\mathrm{H}_{2}, \text { dis }}^{\mathrm{BH}}=4.8 \times 10^{3}$, still much higher than a typical star-forming galaxy which only yields $k_{\mathrm{H}^{-}, \text {det }}^{\mathrm{GAL}} / k_{\mathrm{H}_{2}, \mathrm{dis}}^{\mathrm{GAL}}=44$.

- Ignoring the effect of X-rays, the critical field intensity to suppress $\mathrm{H}_{2}$ formation for the three $\mathrm{DCBH}$ SEDs is $J_{\mathrm{LW}}^{\text {crit }} \approx 22,35$ and 54 , respectively. Note that this is similar to a blackbody radiation field with effective temperature $10^{4} \mathrm{~K},\left(J_{21}^{\text {crit }} \approx 30\right)$, but much lower than for a typical star-forming galaxy spectrum $\left(J_{\mathrm{LW}}^{\mathrm{crit}} \approx 700\right)$. Hence, an emitting $\mathrm{DCBH}$ can trigger the formation of $\mathrm{DCBH}$ in a nearby collapsing gas cloud much more efficiently than galaxies.

- If an XRB produced by previously formed DCBH is present, it may promote $\mathrm{H}_{2}$ formation and increase $J_{\mathrm{LW}}^{\text {crit }}$. For the $N_{\mathrm{H}}=1.3 \times 10^{25} \mathrm{~cm}^{-2}$ model, if the accretion rate density is $\dot{\rho}_{\bullet} \lesssim 10^{-4} M_{\odot} \mathrm{yr}^{-1} \mathrm{Mpc}^{-3}$, XRB plays a negligible role. However, even if $\dot{\rho}_{\bullet}$ reaches the maximum value allowed by the presentday XRB level, i.e. $\sim 0.22 M_{\odot} \mathrm{yr}^{-1} \mathrm{Mpc}^{-3}, J_{\mathrm{LW}}^{\text {crit }}$ only increases to $\approx 80$. For the $N_{\mathrm{H}}=8.0(5.0) \times$ $10^{24} \mathrm{~cm}^{-2}$ SED models, XRB effect is negligible when $\dot{\rho}_{\bullet} \lesssim 10^{-5}\left(10^{-6}\right) M_{\odot} \mathrm{yr}^{-1} \mathrm{Mpc}^{-3}$, and the

the recombination coefficient, $f_{\text {esc }}$ and $\dot{Q}_{\mathrm{H}}$ are the escape fraction and production rate of the ionizing photons respectively. From Pallottini et al. (2014), the typical gas density near the galaxy is $\sim 10-10^{2.5}$ times the mean density of the Universe. However, minihalos and dense filaments may boost this factor. Because of the uncertainties on $n_{\mathrm{H}}$ and $f_{\text {esc }}$, we do not include $r_{\mathrm{B}}$ in our calculation in this subsection. 


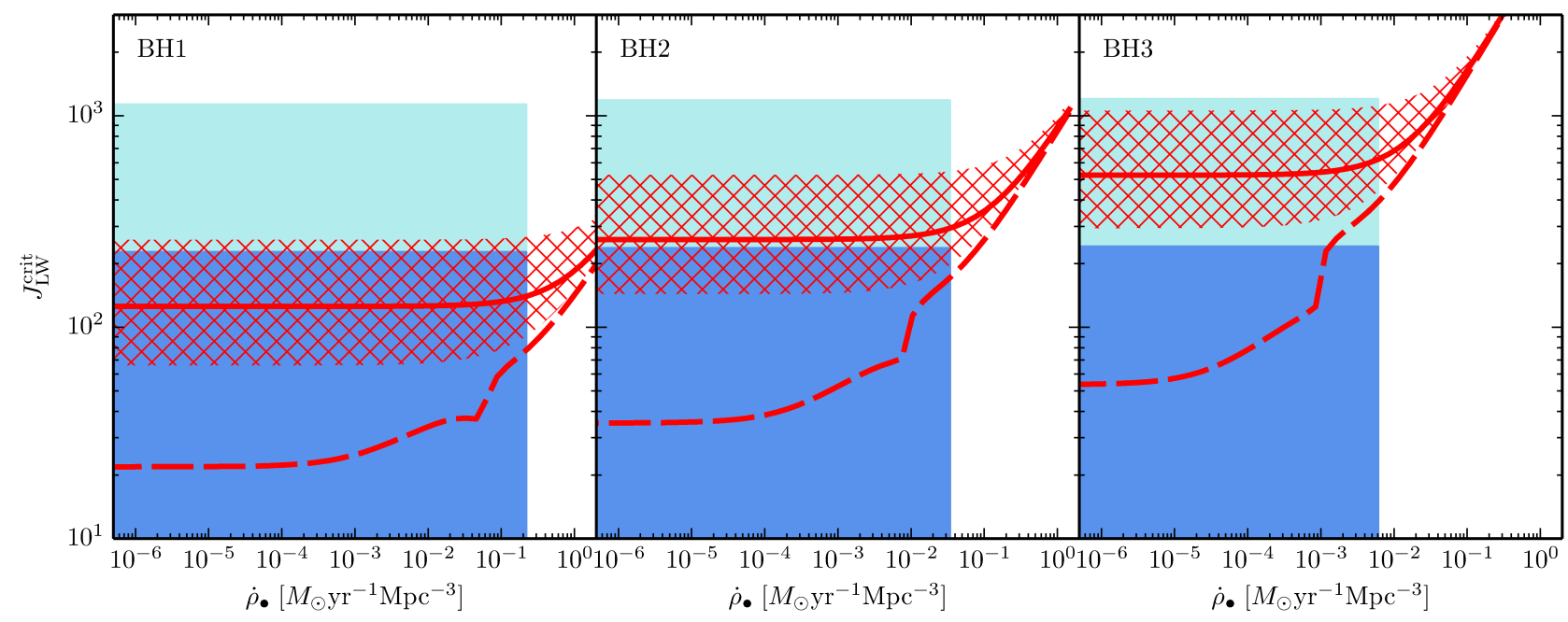

FIG. 9.- The $J_{\mathrm{LW}}^{\text {crit }}$ against $\dot{\rho}_{\bullet}, \mathrm{XRB}$ from first galaxies is included as well. The shaded regions are allowed space by present-day XRB constraints and gravitationally disruption, see Fig. 7 Solid curves correspond to fiducial parameters of first galaxies in Fig. 8 while hatched regions are variance. For reference we also plot the $J_{\mathrm{LW}}^{\text {crit }}$ curves in the absence of XRB from first galaxies by dashed lines.

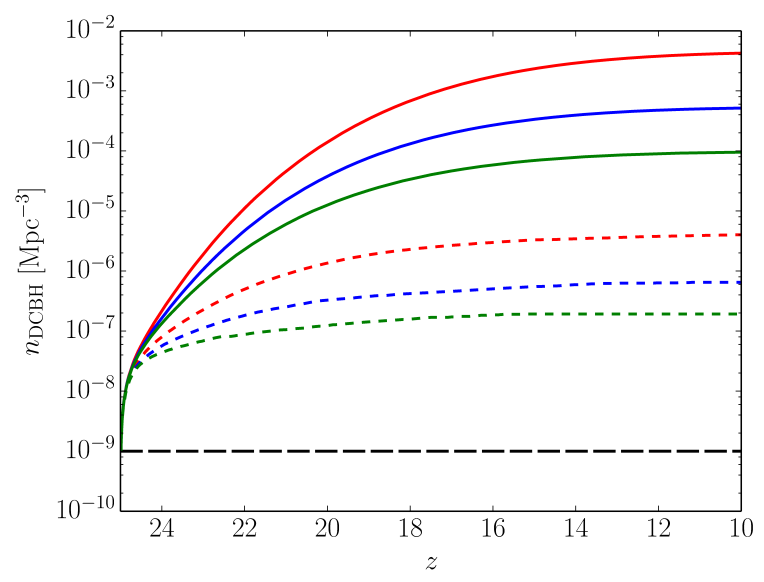

FIG. 10.- The evolution of the number density of DCBHs when different SEDs and critical field strengths are adopted. Solid (dashed) lines are for the critical field strengths without (with) XRB from first galaxies. From top to bottom the three solid lines correspond to BH1 SED with $J_{\mathrm{LW}}^{\text {crit }}=22, \mathrm{BH} 2 \mathrm{SED}$ with $J_{\mathrm{LW}}^{\text {crit }}=35$ and BH3 SED with $J_{\mathrm{LW}}^{\text {crit }}=54$ respectively. The three dashed lines correspond to BH1 SED with $J_{\mathrm{LW}}^{\mathrm{crit}}=130$, BH2 SED with $J_{\mathrm{LW}}^{\text {crit }}=260$ and BH3 SED with $J_{\mathrm{LW}}^{\text {crit }}=520$ respectively, see Fig. 9 The horizontal dashed line refers to the approximate number density of observed SMBHs at $z \gtrsim 6, n_{\mathrm{SMBH}}(z \gtrsim 6) \sim 10^{-9} \mathrm{Mpc}^{-3}$.
$J_{\mathrm{LW}}^{\text {crit }}$ increases to $\approx 170(390)$ at the maximum $\dot{\rho} \bullet$ allowed by the present-day XRB level, which is $\approx 0.034(0.006) M_{\odot} \mathrm{yr}^{-1} \mathrm{Mpc}^{-3}$. However, if the additional but uncertain XRB contribution from first galaxies is included, it may modify the result by factors in the range $\sim 2-20$.

Finally, it is worth noting that several works, for instance Shang et al. (2010), Latif et al. (2014) and Latif et al. (2015), have shown that the $J_{\mathrm{LW}}^{\text {crit }}$ derived from 3D simulations is almost $\sim 10-100$ times higher than from one-zone calculations, "due to the inability of one-zone models to simulate shocks, collapse dynamics and hydrodynamical effects" (Latif et al. 2015). Moreover, they also found that the $J_{\mathrm{LW}}^{\mathrm{crit}}$ varies from halo to halo. Under these circumstances, we might be significantly underestimating $J_{\mathrm{LW}}^{\mathrm{crit}}$. Further numerical work will be necessary to clarify this point.

B.Y. is supported by the CAS Pioneer Hundred Talents (Young Talents) Program. F.P. acknowledges the NASA-ADAP grant MA160009. This work is supported in part by the Grant-in-Aid from the Ministry of Education, Culture, Sports, Science and Technology (MEXT) of Japan (25287040 KO).

\section{REFERENCES}

Abel, T., Anninos, P., Zhang, Y., \& Norman, M. L. 1997, New A, 2,181

Abgrall, H., Le Bourlot, J., Pineau Des Forets, G., et al. 1992, A\&A, 253, 525

Abgrall, H., \& Roueff, E. 1989, A\&AS, 79, 313

Agarwal, B., Dalla Vecchia, C., Johnson, J. L., Khochfar, S., \& Paardekooper, J.-P. 2014, MNRAS, 443, 648

Agarwal, B., Davis, A. J., Khochfar, S., Natarajan, P., \& Dunlop, J. S. 2013, MNRAS, 432, 3438

Agarwal, B., Johnson, J. L., Zackrisson, E., et al. 2016a, MNRAS, 460,4003

Agarwal, B., Khochfar, S., Johnson, J. L., et al. 2012, MNRAS, 425,2854

Agarwal, B., Smith, B., Glover, S., Natarajan, P., \& Khochfar, S. 2016b, MNRAS, 459, 4209

Alvarez, M. A., Wise, J. H., \& Abel, T. 2009, ApJ, 701, L133
Aykutalp, A., Wise, J. H., Spaans, M., \& Meijerink, R. 2014, ApJ, 797, 139

Balucinska-Church, M., \& McCammon, D. 1992, ApJ, 400, 699

Barkana, R., \& Loeb, A. 2001, Phys. Rep., 349, 125

Basu-Zych, A. R., Lehmer, B. D., Hornschemeier, A. E., et al. 2013, ApJ, 762, 45

Begelman, M. C. 2010, MNRAS, 402, 673

Begelman, M. C., Rossi, E. M., \& Armitage, P. J. 2008, MNRAS, 387, 1649

Begelman, M. C., Volonteri, M., \& Rees, M. J. 2006, MNRAS, 370,289

Binney, J., \& Tremaine, S. 1987, Galactic dynamics, 747

Bouwens, R. J., Illingworth, G. D., Oesch, P. A., et al. 2015a, ApJ, 811, 140

-.2015b, ApJ, 803, 34

Bowler, R. A. A., McLure, R. J., Dunlop, J. S., et al. 2016, ArXiv e-prints, arXiv:1609.00727 
Bromm, V., Coppi, P. S., \& Larson, R. B. 2002, ApJ, 564, 23

Bromm, V., \& Loeb, A. 2003, ApJ, 596, 34

Choi, J.-H., Shlosman, I., \& Begelman, M. C. 2015, MNRAS, 450, 4411

Chon, S., Hirano, S., Hosokawa, T., \& Yoshida, N. 2016, ApJ, 832,134

Dijkstra, M., Ferrara, A., \& Mesinger, A. 2014, MNRAS, 442, 2036

Dijkstra, M., Haiman, Z., Mesinger, A., \& Wyithe, J. S. B. 2008, MNRAS, 391, 1961

Eisenstein, D. J., \& Hu, W. 1998, ApJ, 496, 605

Fan, X., Strauss, M. A., Schneider, D. P., et al. 2003, AJ, 125 , 1649

Ferrara, A., Haardt, F., \& Salvaterra, R. 2013, MNRAS, 434, 2600

Ferrara, A., Salvadori, S., Yue, B., \& Schleicher, D. 2014 , MNRAS, 443, 2410

Furlanetto, S. R. 2006, MNRAS, 371, 867

Galli, D., \& Palla, F. 1998, A\&A, 335, 403

Giocoli, C., Moreno, J., Sheth, R. K., \& Tormen, G. 2007, MNRAS, 376, 977

Glover, S. C. O. 2015, MNRAS, 451, 2082

-. 2016, ArXiv e-prints, arXiv:1610.05679

Glover, S. C. O., \& Abel, T. 2008, MNRAS, 388, 1627

Glover, S. C. O., \& Brand, P. W. J. L. 2001, MNRAS, 321, 385

Glover, S. C. O., \& Jappsen, A. 2007, ApJ, 666, 1

Habouzit, M., Volonteri, M., Latif, M., Dubois, Y., \& Peirani, S. 2016, MNRAS, 463, 529

Heger, A., Fryer, C. L., Woosley, S. E., Langer, N., \& Hartmann, D. H. 2003, ApJ, 591, 288

Hirata, C. M., \& Padmanabhan, N. 2006, MNRAS, 372, 1175

Hollenbach, D., \& McKee, C. F. 1979, ApJS, 41, 555

Hosokawa, T., Omukai, K., Yoshida, N., \& Yorke, H. W. 2011, Science, 334, 1250

Inayoshi, K., \& Omukai, K. 2011, MNRAS, 416, 2748

-. 2012, MNRAS, 422, 2539

Inayoshi, K., Omukai, K., \& Tasker, E. 2014, MNRAS, 445, L109

Inayoshi, K., \& Tanaka, T. L. 2015, MNRAS, 450, 4350

Inayoshi, K., Visbal, E., \& Kashiyama, K. 2015, MNRAS, 453, 1692

Jeon, M., Pawlik, A. H., Greif, T. H., et al. 2012, ApJ, 754, 34

Jiang, L., Fan, X. Vestergaard, M., et al. 2007, AJ, 134, 1150

Johnson, J. L., Whalen, D. J., Agarwal, B., Paardekooper, J.-P., \& Khochfar, S. 2014, MNRAS, 445, 686

Johnson, J. L., Whalen, D. J., Li, H., \& Holz, D. E. 2013, ApJ, 771,116

Koushiappas, S. M., Bullock, J. S., \& Dekel, A. 2004, MNRAS, 354,292

Kurk, J. D., Walter, F., Fan, X., et al. 2007, ApJ, 669, 32

Latif, M. A., Bovino, S., Grassi, T., Schleicher, D. R. G., \& Spaans, M. 2015, MNRAS, 446, 3163

Latif, M. A., Bovino, S., Van Borm, C., et al. 2014, MNRAS, 443, 1979

Latif, M. A., \& Ferrara, A. 2016, PASA, 33, e051

Latif, M. A., Schleicher, D. R. G., \& Hartwig, T. 2016, MNRAS, 458,233

Latif, M. A., Schleicher, D. R. G., Schmidt, W., \& Niemeyer, J. 2013a, MNRAS, 433, 1607

Latif, M. A., Schleicher, D. R. G., Schmidt, W., \& Niemeyer, J. C. 2013b, MNRAS, 436, 2989

Lehmer, B. D., Basu-Zych, A. R., Mineo, S., et al. 2016, ApJ, 825,7

Leitherer, C., Ortiz Otálvaro, P. A., Bresolin, F., et al. 2010, ApJS, 189, 309

Leitherer, C., Schaerer, D., Goldader, J. D., et al. 1999, ApJS, 123,3

Lepp, S., \& Shull, J. M. 1984, ApJ, 280, 465

Lodato, G., \& Natarajan, P. 2006, MNRAS, 371, 1813

Madau, P., \& Dickinson, M. 2014, ARA\&A, 52, 415

Martin, P. G., Schwarz, D. H., \& Mandy, M. E. 1996, ApJ, 461, 265

Maselli, A., Ferrara, A., \& Ciardi, B. 2003, MNRAS, 345, 379
Mayer, L., Fiacconi, D., Bonoli, S., et al. 2015, ApJ, 810, 51

Mayer, L., Kazantzidis, S., Escala, A., \& Callegari, S. 2010,

Nature, 466, 1082

Moretti, A., Vattakunnel, S., Tozzi, P., et al. 2012, A\&A, 548, A87

Morganson, E., De Rosa, G., Decarli, R., et al. 2012, AJ, 143, 142

Mortlock, D. J., Warren, S. J., Venemans, B. P., et al. 2011,

Nature, 474, 616

Omukai, K. 2000, ApJ, 534, 809

-. 2001, ApJ, 546, 635

Omukai, K., Hosokawa, T., \& Yoshida, N. 2010, ApJ, 722, 1793

Omukai, K., \& Nishi, R. 1998, ApJ, 508, 141

Omukai, K., Tsuribe, T., Schneider, R., \& Ferrara, A. 2005, ApJ, 626,627

Pacucci, F., \& Ferrara, A. 2015, MNRAS, 448, 104

Pacucci, F., Ferrara, A., Grazian, A., et al. 2016, MNRAS, 459, 1432

Pacucci, F., Ferrara, A., Volonteri, M., \& Dubus, G. 2015a, MNRAS, 454, 3771

Pacucci, F., Mesinger, A., Mineo, S., \& Ferrara, A. 2014, MNRAS, 443, 678

Pacucci, F., Pallottini, A., Ferrara, A., \& Gallerani, S. 2017, MNRAS, 468, L77

Pacucci, F., Volonteri, M., \& Ferrara, A. 2015b, MNRAS, 452, 1922

Palla, F., Salpeter, E. E., \& Stahler, S. W. 1983, ApJ, 271, 632

Pallottini, A., Gallerani, S., \& Ferrara, A. 2014, MNRAS, 444, L105

Pallottini, A., Ferrara, A., Pacucci, F., et al. 2015, MNRAS, 453, 2465

Planck Collaboration, Ade, P. A. R., Aghanim, N., et al. 2016, A\&A, 594, A13

Regan, J. A., \& Haehnelt, M. G. 2009, MNRAS, 396, 343

Regan, J. A., Johansson, P. H., \& Wise, J. H. 2014, ApJ, 795, 137

-. 2016, MNRAS, 461, 111

Salvaterra, R., Haardt, F. \& Ferrara, A. 2005, MNRAS, 362, L50

Shang, C., Bryan, G. L., \& Haiman, Z. 2010, MNRAS, 402, 1249

Shapiro, P. R., \& Kang, H. 1987, ApJ, 318, 32

Sheth, R. K., Mo, H. J., \& Tormen, G. 2001, MNRAS, 323, 1

Sheth, R. K., \& Tormen, G. 1999, MNRAS, 308, 119

Shull, J. M., \& van Steenberg, M. E. 1985, ApJ, 298, 268

Smidt, J., Wiggins, B. K., \& Johnson, J. L. 2016, ApJ, 829, L6

Smith, A., Bromm, V., \& Loeb, A. 2016, MNRAS, 460, 3143

Sobral, D., Matthee, J., Darvish, B., et al. 2015, ApJ, 808, 139

Stacy, A., \& Bromm. V. 2007, MNRAS, 382, 229

Stecher, T. P., \& Williams, D. A. 1967, ApJ, 149, L29

Sugimura, K., Coppola, C. M., Omukai, K., Galli, D., \& Palla, F. 2016, MNRAS, 456, 270

Sugimura, K., Omukai, K., \& Inoue, A. K. 2014, MNRAS, 445, 544

Tanaka, T., Perna, R., \& Haiman, Z. 2012, MNRAS, 425, 2974

Umeda, H., Hosokawa, T., Omukai, K., \& Yoshida, N. 2016, ApJ, 830, L34

Valdés, M.. \& Ferrara, A. 2008, MNRAS, 387, L8

Valiante, R., Schneider, R., Volonteri, M., \& Omukai, K. 2016, MNRAS, 457, 3356

Vázquez, G. A., \& Leitherer, C. 2005, ApJ, 621, 695

Venemans, B. P., Findlay, J. R., Sutherland, W. J., et al. 2013, ApJ, 779, 24

Visbal, E., Haiman, Z., \& Bryan, G. L. 2014a, MNRAS, 445, 1056

- 2014b, MNRAS, 442, L100

Visbal, E., Haiman, Z., Terrazas, B., Bryan, G. L., \& Barkana, R. 2014c, MNRAS, 445, 107

Volonteri, M. 2010, A\&A Rev., 18, 279

Wolcott-Green, J., Haiman, Z., \& Bryan, G. L. 2011, MNRAS, 418,838

-. 2017, MNRAS, arXiv:1609.02142

Wu, X.-B., Wang, F., Fan, X., et al. 2015, Nature, 518, 512

Yoshida, N., Omukai, K., Hernquist, L., \& Abel, T. 2006, ApJ, 652,6

Yue, B., Ferrara, A., Salvaterra, R., Xu, Y., \& Chen, X. 2014, MNRAS, 440, 1263

\section{APPENDIX}

\section{A. THE OPTICAL DEPTHS FOR $\mathrm{H}_{2}, \mathrm{H}^{-}$, AND $\mathrm{H}_{2}^{+}$}

Here we check the validity of the optically thin assumption made for the $\mathrm{H}^{-}$detachment radiation and for the $\mathrm{H}_{2}^{+}$ dissociation radiation. For $\mathrm{H}^{-}$, the mean optical depth of the gas cloud is

$$
\left\langle\tau_{\mathrm{H}^{-}, \text {det }}\right\rangle=\lambda_{J} / 2\left\langle\sigma_{\mathrm{H}^{-}, \text {det }}\right\rangle n_{\mathrm{H}^{-}},
$$




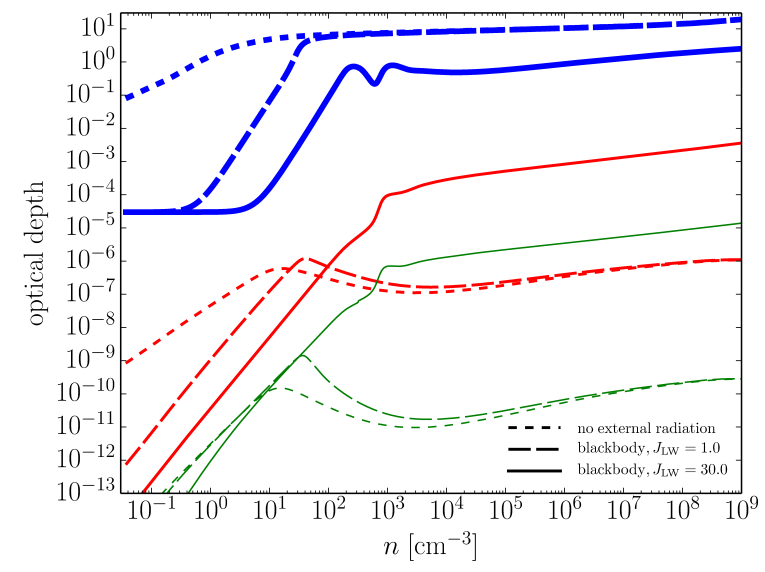

FIG. 11. - The optical depth against $n$, thick lines are $-\log \left(f_{\mathrm{sh}}\right)$ for $\mathrm{H}_{2}$ self-shielding, medium and thin lines are for the $\mathrm{H}^{-}$and $\mathrm{H}_{2}^{+}$ respectively.

where the mean cross-section is

$$
\left\langle\sigma_{\mathrm{H}^{-}, \mathrm{det}}\right\rangle=k_{\mathrm{H}^{-}, \operatorname{det}}\left(\int_{\nu_{0.755}}^{\nu_{13.6}} \frac{4 \pi J(\nu)}{h \nu} d \nu\right)^{-1}
$$

This mean cross-section only depends on the spectral shape of the external radiation field, however is independent of its strength which appears in both numerator and denominator. For the BB spectrum, $\left\langle\sigma_{\mathrm{H}^{-} \text {,det }}\right\rangle=2.8 \times 10^{-17} \mathrm{~cm}^{2}$. Similarly, we can also calculate the mean cross-section for $\mathrm{H}_{2}^{+}$, for which we get $\left\langle\sigma_{\mathrm{H}_{2}^{+} \text {,dis }}\right\rangle=7.0 \times 10^{-19} \mathrm{~cm}^{-2}$, and the corresponding mean optical depth $\left\langle\tau_{\mathrm{H}_{2}^{+}, \mathrm{dis}}\right\rangle$.

In Fig. 11 we plot $\left\langle\tau_{\mathrm{H}^{-}, \text {det }}\right\rangle,\left\langle\tau_{\mathrm{H}_{2}^{+} \text {,dis }}\right\rangle$ and $-\log \left(f_{\mathrm{sh}}\right)$ (considered as the mean optical depth to LW radiation) respectively against the $n$ for different external radiation field strengths. This figure shows that, since the optical depth to $\mathrm{H}$ ? detachment radiation and to $\mathrm{H}+2$ dissociation radiation are always much smaller, it is then safe to ignore them. On the other hand, even when $J_{\mathrm{LW}} \gtrsim J_{\mathrm{LW}}^{\text {crit }}$, at $n \gtrsim 10^{3} \mathrm{~cm}^{-3}$ the self-shielding effect to LW radiation is not negligible.

\section{B. PARAMETER WINDOWS FOR ENHANCED DCBH FORMATION PROBABILITY}

In our fiducial model we adopt the following initial setup: $z_{\text {ta }}=30.6$; adiabatic IGM temperature; ionization fraction $10^{-4}, \mathrm{H}_{2}$ fraction $10^{-6}$. In this section we investigate the influence of alternative initial setups on DCBH formation.

We first check that for $\mathrm{BH} 1$ radiation field, using $z_{\mathrm{ta}}=16$ while keeping other parameters fixed to the fiducial ones, we get $J_{\mathrm{LW}}^{\text {crit }} \approx 20$. As for the fiducial setup for which we get $J_{\mathrm{LW}}^{\text {crit }} \approx 22$, we conclude that DCBH formation is insensitive to cosmic epoch at which the process takes place.

We then take into account pre-heating of gas by X-rays and shocks before it collapses. In such case at $z=z_{\text {ta }}$ the gas temperature is higher than the adiabatic IGM temperature. We set the initial gas temperature $T_{0}=30,50,100$ and $1000 \mathrm{~K}$ respectively, keeping other initial setups the same as our fiducial model, and find that $J_{\mathrm{LW}}^{\text {crit }}$ increases with increasing $T_{0}: J_{\mathrm{LW}}^{\text {crit }} \approx 39,49,50$ and 51 for $T_{0}=30,50,100$ and $1000 \mathrm{~K}$ respectively. When $T_{0} \gtrsim 100 \mathrm{~K}$ the $J_{\mathrm{LW}}^{\text {crit }}$ almost does no longer increase. We conclude that ignoring the gas pre-heating may underestimate the critical field intensity by a factor at most $\approx 2$.

Interestingly, we find that in the model with $T_{0}=30 \mathrm{~K}, \mathrm{H}_{2}$ formation and cooling could also be suppressed even when the radiation field strength is smaller than $J_{\mathrm{LW}}^{\text {crit }} \approx 39$, as long as it is in a narrow range $14.5 \lesssim J_{\mathrm{LW}} \lesssim 15.5$, as shown by Fig. 12 where we plot the gas temperature at the final step of our calculations $\left(n \sim 10^{9} \mathrm{~cm}^{-3}\right)$ against $J_{\mathrm{LW}}$. To investigate the physics behind this interesting phenomenon, we look through the evolution track and find that when $J_{\mathrm{LW}}$ is in this range, the gas almost simultaneously reaches the maximum temperature $\sim 10^{4} \mathrm{~K}$ and the critical density for $\mathrm{H}-\mathrm{H}_{2}$ collisional dissociation, $n_{\mathrm{cr}, \mathrm{H}}$ ( see Martin et al. 1996). When gas number density is higher than $n_{\mathrm{cr}, \mathrm{H}}, \mathrm{H}_{2}$ is dissociated via the $\mathrm{H}_{2}+\mathrm{H} \stackrel{\mathrm{k}_{13}}{\longrightarrow} 3 \mathrm{H}$ reaction with reaction rate $k_{13}$ approximately proportional to $\exp \left(-5 \times 10^{4} / T\right)$. It has strong temperature dependence hence the $\mathrm{H}_{2}$ is dissociated very efficiently at the maximum gas temperature, resulting in suppressed $\mathrm{H}_{2}$ cooling. If, however, the $J_{\mathrm{LW}}$ is smaller than $\sim 14.5$, the gas cools earlier and never has a chance to reach the temperature $\sim 10^{4} \mathrm{~K}$, and the collision dissociation is not efficient because of the low temperature; or if the $J_{\mathrm{LW}}$ is larger than $\sim 15.5$, the radiation reduces (but not fully suppresses) $\mathrm{H}_{2}$ cooling and the gas temperature reaches $10^{4} \mathrm{~K}$ before the gas density reaches $n_{\mathrm{cr}, \mathrm{H}}$, therefore the $\mathrm{H}_{2}$ cannot be dissociated efficiently via $\mathrm{H}-\mathrm{H}_{2}$ collisions because of the low density, and then the gas temperature decreases soon. The same phenomenon also exists in the $T_{0}=50 \mathrm{~K}$ model, except that the feasible field intensity range is even narrower. However, it does not exist in our fiducial model where $T_{0}=19.8 \mathrm{~K}$, and the model with $T_{0}>50 \mathrm{~K}$. 


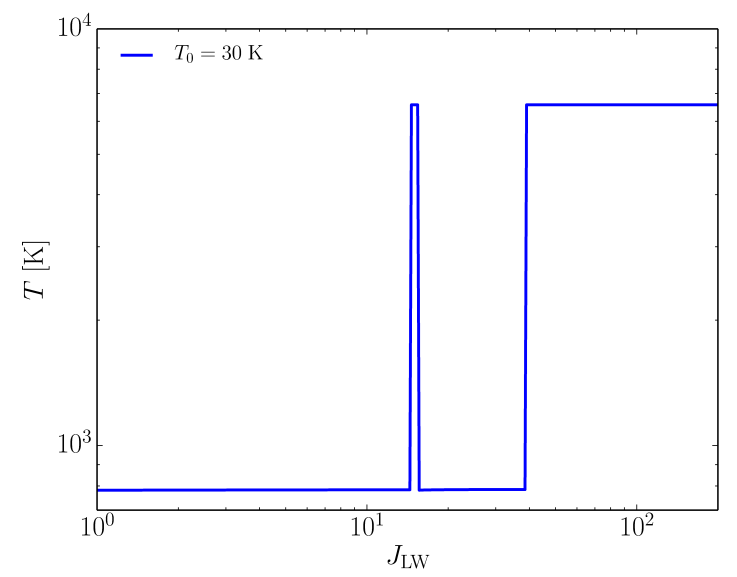

FIG. 12. - For BH1 radiation field, the gas temperature at $n \sim 10^{9} \mathrm{~cm}^{-3}$ vs. $J_{\mathrm{LW}}$ when gas has initial temperature $T_{0}=30 \mathrm{~K}$.

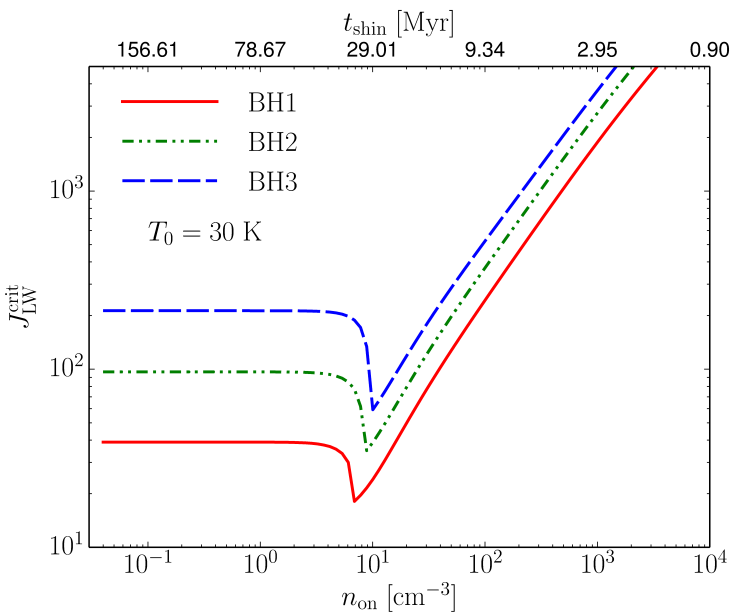

Fig. 13.- Same to Fig. 5 however $T_{0}=30 \mathrm{~K}$.

Although the physics mechanism is interesting, the above phenomenon may hardly occur in more realistic cases in which the strength of the external radiation varies with time. However, this mechanism could work in other situations and decrease the critical field intensity, see below.

We re-calculate the $J_{\mathrm{LW}}^{\text {crit }}-n_{\text {on }}$ relation for $T_{0}=30 \mathrm{~K}$, the results are plotted in Fig. 13, We find the $J_{\mathrm{LW}}^{\text {crit }}$ does not increase monotonically with $n_{\mathrm{on}}$. Instead, for BH1 (BH2, BH3) radiation field when $2 \mathrm{~cm}^{-3} \lesssim n_{\text {on }} \lesssim 15 \mathrm{~cm}^{-3}$ $\left(2 \mathrm{~cm}^{-3} \lesssim n_{\mathrm{on}} \lesssim 25 \mathrm{~cm}^{-3}, 2 \mathrm{~cm}^{-3} \lesssim n_{\mathrm{on}} \lesssim 35 \mathrm{~cm}^{-3}\right), J_{\mathrm{LW}}^{\text {crit }}$ is even smaller than models where the external radiation field irradiates the collapsing gas cloud longer. For example, if the external radiation is switched on at $\sim 7 \mathrm{~cm}^{-3}$, $\mathrm{H}_{2}$ suppression is achieved with an intensity as low as $J_{\mathrm{LW}}^{\text {crit }} \sim 18$ for BH1 radiation field. We clarify here that the $J_{\mathrm{LW}}^{\text {crit }}$ is not the left boundary of the narrow peak in Fig. 12, instead it is the left boundary of the plateau, i.e. for all $J_{\mathrm{LW}} \geq J_{\mathrm{LW}}^{\text {crit }}$ the $\mathrm{H}_{2}$ formation and cooling will be suppressed.

The physics of this phenomenon is same to the above. Given $J_{\mathrm{LW}}=30$, in Fig. 14we plot the the temperature tracks for $n_{\mathrm{on}}=(1,10,20) \mathrm{cm}^{-3}$. We also plot the $T-n_{\mathrm{cr}, \mathrm{H}}$ curve in the same panel. Note that $J_{\mathrm{LW}}=30$ is smaller than the $J_{\mathrm{LW}}^{\text {crit }}$ for $n_{\mathrm{on}}=1$ and $20 \mathrm{~cm}^{-3}$, but larger than the $J_{\mathrm{LW}}^{\text {crit }}$ for $n_{\mathrm{on}}=10 \mathrm{~cm}^{-3}$. As seen from Fig. 14 for $n_{\mathrm{on}}=1 \mathrm{~cm}^{-3}$ and $20 \mathrm{~cm}^{-3}$ cases, the temperature track and the $T-n_{\mathrm{cr}, \mathrm{H}}$ curve intersect at $T \sim 500 \mathrm{~K}$. However for the $n_{\mathrm{on}}=10$ $\mathrm{cm}^{-3}$ case, the intersection is near $10000 \mathrm{~K}$. Recall that at $\gtrsim n_{\mathrm{cr}, \mathrm{H}}$ the reaction rate $k_{13}$ approximately proportional to $\exp \left(-5 \times 10^{4} / T\right)$. Hence, only if the temperature track and the $T-n_{\mathrm{cr}, \mathrm{H}}$ curve intersect at high temperatures the $\mathrm{H}_{2}$ can be rapidly dissociated by $\mathrm{H}-\mathrm{H}_{2}$ collisions, see the colored segment of each curve where the $k_{13}$ is shown. In such a situation the $\mathrm{H}_{2}$ formation and cooling suppression by a weaker external radiation is possible. Moreover, when $n_{\mathrm{on}} \gtrsim 100 \mathrm{~cm}^{-3}$, the $J_{\mathrm{LW}}^{\text {crit }}$ increases rapidly because otherwise the previously formed $\mathrm{H}_{2}$ has already cooled the gas to a low temperatures. We note that from $n \sim 2 \mathrm{~cm}^{-3}$ to $\sim 15 \mathrm{~cm}^{-3}$ only about $\sim 35 \mathrm{Myr}$ time elapses. It is still a short time-scale opportunity for DCBH to form in a weaker external radiation field.

\section{REACTION RATES AND COOLING FUNCTIONS}

Tables 3 - 5 list the rates of reactions, and heating and cooling functions adopted in this work. 


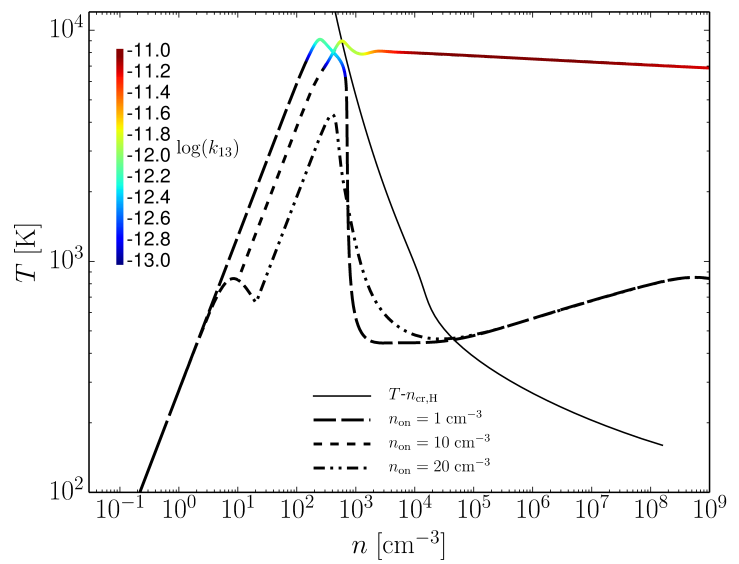

FIG. 14. - The temperature track if the external BH1 radiation field switched on at total number density 1,10 and $20 \mathrm{~cm}^{-3}$ respectively. In the temperature we color the segment where the $\mathrm{H}_{2}$ collisional dissociation rate $k_{13}>10^{-13} \mathrm{~cm}^{3} \mathrm{~s}^{-1}$. In the same panel we also plot the $T-n_{\mathrm{cr}, \mathrm{H}}$ curve.

TABLE 3

RATES OF REACTIONS ADOPTED IN THIS WORK.

\begin{tabular}{|c|c|c|c|c|}
\hline Ordinal & Reaction & Rate $^{\mathrm{a}, \mathrm{b}}$ & & units \\
\hline (1) & $\mathrm{H}+\mathrm{e}^{-} \rightarrow \mathrm{H}^{+}+2 \mathrm{e}^{-}$ & $\begin{aligned} k_{1}=\exp [ & -32.71396786375 \\
& +13.53655609057\left(\ln T_{\mathrm{eV}}\right) \\
& -5.739328757388\left(\ln T_{\mathrm{eV}}\right)^{2} \\
& +1.563154982022\left(\ln T_{\mathrm{eV}}\right)^{3} \\
& -0.2877056004391\left(\ln T_{\mathrm{eV}}\right)^{4} \\
& +0.03482559773736999\left(\ln T_{\mathrm{eV}}\right)^{5} \\
& -0.00263197617559\left(\ln T_{\mathrm{eV}}\right)^{6} \\
& +0.0001119543953861\left(\ln T_{\mathrm{eV}}\right)^{7} \\
& \left.-2.039149852002 \times 10^{-6}\left(\ln T_{\mathrm{eV}}\right)^{8}\right]\end{aligned}$ & & $\mathrm{cm}^{3} \mathrm{~s}^{-1}$ \\
\hline (2) & $\mathrm{He}+\mathrm{e}^{-} \rightarrow \mathrm{He}^{+}+2 \mathrm{e}^{-}$ & $\begin{aligned} k_{2}=\exp [ & -44.09864886561001 \\
& +23.91596563469\left(\ln T_{\mathrm{eV}}\right) \\
& -10.75323019821\left(\ln T_{\mathrm{eV}}\right)^{2} \\
& +3.058038757198\left(\ln T_{\mathrm{eV}}\right)^{3} \\
& -0.5685118909884001\left(\ln T_{\mathrm{eV}}\right)^{4} \\
& +0.06795391233790001\left(\ln T_{\mathrm{eV}}\right)^{5} \\
& -0.005009056101857001\left(\ln T_{\mathrm{eV}}\right)^{6} \\
& +0.0002067236157507\left(\ln T_{\mathrm{eV}}\right)^{7} \\
& \left.-3.649161410833 \times 10^{-6}\left(\ln T_{\mathrm{eV}}\right)^{8}\right]\end{aligned}$ & & $\mathrm{cm}^{3} \mathrm{~s}^{-1}$ \\
\hline (3) & $\mathrm{H}^{+}+\mathrm{e}^{-} \rightarrow \mathrm{H}+\gamma$ & $\begin{aligned} k_{3}=\exp [ & -28.61303380689232 \\
& -0.7241125657826851\left(\ln T_{\mathrm{eV}}\right) \\
& -0.02026044731984691\left(\ln T_{\mathrm{eV}}\right)^{2} \\
& -0.002380861877349834\left(\ln T_{\mathrm{eV}}\right)^{3} \\
& -0.0003212605213188796\left(\ln T_{\mathrm{eV}}\right)^{4} \\
& -0.00001421502914054107\left(\ln T_{\mathrm{eV}}\right)^{5} \\
& +4.989108920299513 \times 10^{-6}\left(\ln T_{\mathrm{eV}}\right)^{6} \\
& +5.755614137575758 \times 10^{-7}\left(\ln T_{\mathrm{eV}}\right)^{7} \\
& -1.856767039775261 \times 10^{-8}\left(\ln T_{\mathrm{eV}}\right)^{8} \\
& \left.-3.071135243196595 \times 10^{-9}\left(\ln T_{\mathrm{eV}}\right)^{9}\right]\end{aligned}$ & & $\mathrm{cm}^{3} \mathrm{~s}^{-1}$ \\
\hline (4) & $\mathrm{He}^{+}+\mathrm{e}^{-} \rightarrow \mathrm{He}+\gamma$ & $\begin{aligned} k_{4}= & 1.54 \times 10^{-9}\left[1+0.3 / \exp \left(8.099328789667 / T_{\mathrm{eV}}\right)\right] / \\
& {\left[\exp \left(40.49664394833662 / T_{\mathrm{eV}}\right) \times T_{\mathrm{eV}}^{1.5}\right] } \\
& +3.92 \times 10^{-13} / T_{\mathrm{eV}}^{0.6353}\end{aligned}$ & & $\mathrm{~cm}^{3} \mathrm{~s}^{-1}$ \\
\hline (5) & $\mathrm{H}+\mathrm{H}^{+} \rightarrow \mathrm{H}_{2}^{+}+\gamma$ & $\begin{array}{l}k_{5}=1.85 \times 10^{-23} T^{1.8} \\
k_{5}=5.81 \times 10^{-16}(T / 56200)^{[-0.6657 \log (T / 56200)]}\end{array}$ & $\begin{array}{l}T \leq 6.7 \times 10^{3} \mathrm{~K} \\
T>6.7 \times 10^{3} \mathrm{~K}\end{array}$ & $\mathrm{~cm}^{3} \mathrm{~s}^{-1}$ \\
\hline (6) & $\mathrm{H}_{2}^{+}+\mathrm{H} \rightarrow \mathrm{H}_{2}+\mathrm{H}^{+}$ & $k_{6}=6.0 \times 10^{-10}$ & & $\mathrm{~cm}^{3} \mathrm{~s}^{-1}$ \\
\hline (7) & $\mathrm{H}+\mathrm{e}^{-} \rightarrow \mathrm{H}^{-}+\gamma$ & $k_{7}=6.775 \times 10^{-15}\left(T_{\mathrm{eV}}\right)^{0.8779}$ & & $\mathrm{~cm}^{3} \mathrm{~s}^{-1}$ \\
\hline (8) & $\mathrm{H}+\mathrm{H}^{-} \rightarrow \mathrm{H}_{2}+\mathrm{e}^{-}$ & $\begin{aligned} k_{8}=1.43 & \times 10^{-9} \\
k_{8}=\exp [ & -20.06913897587003 \\
& +0.2289800603272916\left(\ln T_{\mathrm{eV}}\right) \\
& +0.03599837721023835\left(\ln T_{\mathrm{eV}}\right)^{2} \\
& -0.004555120027032095\left(\ln \left(T_{\mathrm{eV}}\right)^{3}\right.\end{aligned}$ & $\begin{array}{l}T_{\mathrm{eV}} \leq 0.1 \\
T_{\mathrm{eV}}>0.1\end{array}$ & $\mathrm{~cm}^{3} \mathrm{~s}^{-1}$ \\
\hline
\end{tabular}


TABLE 3 - Continued

\begin{tabular}{|c|c|c|c|c|}
\hline Ordinal & Reaction & Rate $^{\mathrm{a}, \mathrm{b}}$ & & units \\
\hline & & $\begin{array}{l}-0.0003105115447124016\left(\ln \left(T_{\mathrm{eV}}\right)^{4}\right. \\
+0.0001073294010367247\left(\ln \left(T_{\mathrm{eV}}\right)^{5}\right. \\
-8.36671960467864 \times 10^{-6}\left(\ln T_{\mathrm{eV}}\right)^{6} \\
+2.238306228891639 \times 10^{-7}\left(\ln \left(T_{\mathrm{eV}}\right)^{7}\right]\end{array}$ & & \\
\hline (9) & $\mathrm{H}_{2}^{+}+\mathrm{e}^{-} \rightarrow 2 \mathrm{H}$ & $\begin{array}{l}k_{9}=1 \times 10^{-8} \\
k_{9}=1.32 \times 10^{-6} T^{-0.76}\end{array}$ & $\begin{array}{l}T \leq 617 \mathrm{~K} \\
T>617 \mathrm{~K}\end{array}$ & $\mathrm{~cm}^{3} \mathrm{~s}^{-1}$ \\
\hline (10) & $\mathrm{H}_{2}^{+}+\mathrm{H}^{-} \rightarrow \mathrm{H}_{2}+\mathrm{H}$ & $k_{10}=5 \times 10^{-6} T^{-0.5}$ & & $\mathrm{~cm}^{3} \mathrm{~s}^{-1}$ \\
\hline$(11)$ & $\mathrm{H}^{-}+\mathrm{H}^{+} \rightarrow 2 \mathrm{H}$ & $k_{11}=6.5 \times 10^{-9} T_{\mathrm{eV}}^{-0.5}$ & & $\mathrm{~cm}^{3} \mathrm{~s}^{-1}$ \\
\hline (12) & $\mathrm{H}_{2}+\mathrm{e}^{-} \rightarrow \mathrm{H}+\mathrm{H}^{-}$ & $k_{12}=2.7 \times 10^{-8} T^{-1.27} \exp (-43000 / T)$ & & $\mathrm{cm}^{3} \mathrm{~s}^{-1}$ \\
\hline (13) & $\mathrm{H}_{2}+\mathrm{H} \rightarrow 3 \mathrm{H}$ & see Martin et al. (1996) & & $\mathrm{cm}^{3} \mathrm{~s}^{-1}$ \\
\hline (14) & $\mathrm{H}_{2}+\mathrm{H}_{2} \rightarrow \mathrm{H}_{2}+2 \mathrm{H}$ & $\begin{array}{l}k_{14, \mathrm{~L}}=1.18 \times 10^{-10} \exp \left(-6.95 \times 10^{4} / T\right) \\
k_{14, \mathrm{H}}=8.125 \times 10^{-8} T^{-1 / 2} \exp \left(-5.2 \times 10^{4} / T\right)\left[1-\exp \left(-6 \times 10^{3} / T\right)\right] \\
\log n_{\mathrm{cr}, \mathrm{H}_{2}}=4.845-1.3 \log \left(T / 10^{4}\right)+1.62\left[\log \left(T / 10^{4}\right)\right]^{2} \\
a=\left(1+n_{\mathrm{H}} / n_{\mathrm{cr}, \mathrm{H}_{2}}\right)^{-1}, k_{14}=k_{14, \mathrm{H}}^{1-a} k_{14, \mathrm{~L}}^{a}\end{array}$ & & $\mathrm{~cm}^{3} \mathrm{~s}^{-1}$ \\
\hline (15) & $\mathrm{H}_{2}+\mathrm{H}^{+} \rightarrow \mathrm{H}_{2}^{+}+\mathrm{H}$ & $\begin{aligned} k_{15}=\exp [ & -24.24914687731536 \\
& +3.400824447095291\left(\ln T_{\mathrm{eV}}\right) \\
& -3.898003964650152\left(\ln T_{\mathrm{eV}}\right)^{2} \\
& +2.045587822403071\left(\ln T_{\mathrm{eV}}\right)^{3} \\
& -0.5416182856220388\left(\ln T_{\mathrm{eV}}\right)^{4} \\
& +0.0841077503763412\left(\ln T_{\mathrm{eV}}\right)^{5} \\
& -0.007879026154483455\left(\ln T_{\mathrm{eV}}\right)^{6} \\
& +0.0004138398421504563\left(\ln T_{\mathrm{eV}}\right)^{7} \\
& \left.-9.36345888928611 \times 10^{-6}\left(\ln T_{\mathrm{eV}}\right)^{8}\right]\end{aligned}$ & & $\mathrm{cm}^{3} \mathrm{~s}^{-1}$ \\
\hline (16) & $\mathrm{H}_{2}+\mathrm{e}^{-} \rightarrow 2 \mathrm{H}+\mathrm{e}^{-}$ & $5.6 \times 10^{-11} \exp (-102124 / T) T^{0.5}$ & & $\mathrm{~cm}^{3} \mathrm{~s}^{-1}$ \\
\hline (17) & $\mathrm{H}^{-}+\mathrm{e}^{-} \rightarrow \mathrm{H}+2 \mathrm{e}^{-}$ & $\begin{aligned} k_{17}=\exp [ & -18.01849334273 \\
& +2.360852208681\left(\ln T_{\mathrm{eV}}\right) \\
& -0.2827443061704\left(\ln T_{\mathrm{eV}}\right)^{2} \\
& +0.01623316639567\left(\ln T_{\mathrm{eV}}\right)^{3} \\
& -0.03365012031362999\left(\ln T_{\mathrm{eV}}\right)^{4} \\
& +0.01178329782711\left(\ln T_{\mathrm{eV}}\right)^{5} \\
& -0.001656194699504\left(\ln T_{\mathrm{eV}}\right)^{6} \\
& +0.0001068275202678\left(\ln T_{\mathrm{eV}}\right)^{7} \\
& \left.-2.631285809207 \times 10^{-6}\left(\ln T_{\mathrm{eV}}\right)^{8}\right]\end{aligned}$ & & $\mathrm{cm}^{3} \mathrm{~s}^{-1}$ \\
\hline (18) & $\mathrm{H}^{-}+\mathrm{H} \rightarrow 2 \mathrm{H}+\mathrm{e}^{-}$ & $\begin{aligned} k_{18}=2.56 & \times 10^{-9} T_{\mathrm{eV}}^{1.78186} \\
k_{18}=\exp [ & -20.37260896533324 \\
& +1.139449335841631\left(\ln T_{\mathrm{eV}}\right) \\
& -0.1421013521554148\left(\ln T_{\mathrm{eV}}\right)^{2} \\
& +0.00846445538663\left(\ln T_{\mathrm{eV}}\right)^{3} \\
& -0.0014327641212992\left(\ln T_{\mathrm{eV}}\right)^{4} \\
& +0.0002012250284791\left(\ln T_{\mathrm{eV}}\right)^{5} \\
& +0.0000866396324309\left(\ln T_{\mathrm{eV}}\right)^{6} \\
& -0.00002585009680264\left(\ln T_{\mathrm{eV}}\right)^{7} \\
& +2.4555011970392 \times 10^{-6}\left(\ln T_{\mathrm{eV}}\right)^{8} \\
& \left.-8.06838246118 \times 10^{-8}\left(\ln T_{\mathrm{eV}}\right)^{9}\right]\end{aligned}$ & $\begin{array}{l}T_{\mathrm{eV}} \leq 0.1 \\
T_{\mathrm{eV}}>0.1\end{array}$ & $\mathrm{~cm}^{3} \mathrm{~s}^{-1}$ \\
\hline (19) & $\mathrm{H}^{-}+\mathrm{H}^{+} \rightarrow \mathrm{H}_{2}^{+}+\mathrm{e}^{-}$ & $\begin{array}{l}k_{19}=4 \times 10^{-4} T^{-1.4} \exp (-15100 / T) \\
k_{19}=1 \times 10^{-8} T^{-0.4}\end{array}$ & $\begin{array}{l}T \leq 10^{4} \mathrm{~K} \\
T>10^{4} \mathrm{~K}\end{array}$ & $\mathrm{~cm}^{3} \mathrm{~s}^{-1}$ \\
\hline$(20)$ & $\mathrm{H}+\mathrm{H}+\mathrm{H} \rightarrow \mathrm{H}_{2}+\mathrm{H}$ & $k_{20}=5.5 \times 10^{-29} T^{-1}$ & & $\mathrm{~cm}^{6} \mathrm{~s}^{-1}$ \\
\hline (21) & $\mathrm{H}+\mathrm{H}+\mathrm{H}_{2} \rightarrow \mathrm{H}_{2}+\mathrm{H}_{2}$ & $k_{21}=k_{20} / 8$ & & $\mathrm{~cm}^{6} \mathrm{~s}^{-1}$ \\
\hline$(22)$ & $\mathrm{H}_{2}+\gamma \rightarrow 2 \mathrm{H}$ & see Eq. 14, 17 \& 18) & & $\mathrm{s}^{-1}$ \\
\hline (23) & $\mathrm{H}^{-}+\gamma \rightarrow \mathrm{H}+\mathrm{e}^{-}$ & see Eq. 21 22 \& 23: & & $\mathrm{s}^{-1}$ \\
\hline$(24)$ & $\mathrm{H}_{2}^{+}+\gamma \rightarrow \mathrm{H}+\mathrm{H}^{+}$ & see Eq. 24 25 \& 26) & & $\mathrm{s}^{-1}$ \\
\hline
\end{tabular}


TABLE 4 - Continued

\begin{tabular}{lll}
\hline \hline Oridinal & Processes & Heating functions ${ }^{\mathrm{d}}\left[\mathrm{erg} \mathrm{cm}^{-3} \mathrm{~s}^{-1}\right]$
\end{tabular}

${ }^{\mathrm{a}} T_{\mathrm{eV}}$ and $\mathrm{T}$ are temperature in units $\mathrm{eV}$ and $\mathrm{K}$ respectively.

b Reaction (12) is from Glover \& Abel (2008); reaction (14) is from Omukai (2000); reactions (20) and (21) are from Palla et al. (1983); others are from Shang et al. (2010); original references are found in these cited papers.

TABLE 4

HEATING FUNCTIONS USED IN THIS WORK.

\begin{tabular}{lll}
\hline \hline Oridinal & Processes & Heating functions ${ }^{\mathrm{d}}\left[\mathrm{erg} \mathrm{cm}^{-3} \mathrm{~s}^{-1}\right]$ \\
\hline$(1)^{\mathrm{e}}$ & 2 body $\mathrm{H}_{2}$ formation & $\mathcal{H}_{2 b}=1.6 \times 10^{-12}\left\{k_{8} n_{\mathrm{H}}-n_{\mathrm{H}}\left[3.53\left(1+n_{\mathrm{cr}} / n_{\mathrm{H}}\right)^{-1}\right]+k_{6} n_{\mathrm{H}_{2}} n_{\mathrm{H}}\left[1.83\left(1+n_{\mathrm{cr}} / n_{\mathrm{H}}\right)^{-1}\right]\right\}$ \\
$(2)$ & 3 body $\mathrm{H}_{2}$ formation & $\mathcal{H}_{3 b}=1.6 \times 10^{-12} \times 4.48\left(k_{20} n_{\mathrm{H}} n_{\mathrm{H}} n_{\mathrm{H}}+k_{21} n_{\mathrm{H}} n_{\mathrm{H}} n_{\mathrm{H}_{2}}\right)$ \\
\hline & \\
All from Yoshida et al. (2006) $),$ original references are found therein. \\
$n_{\mathrm{cr}}=10^{6} T^{-1 / 2}\left\{1.6 y_{\mathrm{H}} \exp \left[-\left(\frac{400}{T}\right)^{2}\right]+1.4 y_{\mathrm{H}_{2}} \exp \left[-\left(\frac{12000}{T+1200}\right)\right]\right\}^{-1}, y_{\mathrm{H}}$ and $y_{\mathrm{H}_{2}}$ are $\mathrm{H}$ and $\mathrm{H}_{2}$ number fractions respectively.
\end{tabular}

TABLE 5

COOLING FUNCTIONS USED IN THIS WORK.

\begin{tabular}{|c|c|c|}
\hline Oridinal & Processes & Cooling functions ${ }^{\mathrm{f}, \mathrm{g}}\left[\mathrm{erg} \mathrm{cm}^{-3} \mathrm{~s}^{-1}\right]$ \\
\hline (1) & collisional excitation & $\begin{aligned} \Lambda_{\mathrm{H}_{2}, \mathrm{n}_{\mathrm{H}} \rightarrow 0}= & \operatorname{dex}\left[-103.0+97.59 \log T-48.05(\log T)^{2}+10.80(\log T)^{3}-0.9032(\log T)^{4}\right] n_{\mathrm{H}} n_{\mathrm{H}_{2}} \\
\Lambda_{\mathrm{H}_{2}, \mathrm{LTE}}= & \left\{6.7 \times 10^{-19} \exp \left(-\frac{5.86}{T_{3}}\right)+1.6 \times 10^{-18} \exp \left(-\frac{11.7}{T_{3}}\right)\right. \\
& \left.+\frac{9.5 \times 10^{-22} T_{3}^{3.76}}{1+0.12 T_{3}^{2.1}} \exp \left[-\left(\frac{0.13}{T_{3}}\right)^{3}\right]+3 \times 10^{-24} \exp \left(-\frac{0.51}{T_{3}}\right)\right\} n_{\mathrm{H}_{2}} \\
\Lambda_{\mathrm{H}_{2}}^{\text {col-exc }}= & \frac{\Lambda_{\mathrm{H}_{2}, \mathrm{LTE}}}{\left(1+\Lambda_{\mathrm{H}_{2}, \mathrm{LTE}} / \Lambda_{\mathrm{H}_{2}, \mathrm{n}_{\mathrm{H}} \rightarrow 0}\right)}\end{aligned}$ \\
\hline$(2)$ & collisional dissociation & $\Lambda_{\mathrm{H}_{2}}^{\text {col-dis }}=7.2 \times 10^{-12}\left(k_{13} n_{\mathrm{H}}+k_{14} n_{\mathrm{H}_{2}}\right) n_{\mathrm{H}_{2}}$ \\
\hline (3) & recombination & $\begin{array}{l}\Lambda_{\mathrm{H}}^{\mathrm{rec}}=8.7 \times 10^{-27} T^{0.5} T_{3}^{-0.2}\left(1+T_{6}^{0.7}\right)^{-1} n_{\mathrm{e}} n_{\mathrm{H}^{+}} \\
\Lambda_{\mathrm{He}}^{\mathrm{rec}}=1.55 \times 10^{-26} T^{0.3647} n_{\mathrm{e}} n_{\mathrm{He}^{+}}\end{array}$ \\
\hline (4) & collisional ionization & $\begin{array}{l}\Lambda_{\mathrm{H}}^{\text {col-ion }}=1.27 \times 10^{-21} T^{1 / 2}\left(1+T_{5}^{0.5}\right)^{-1} \exp \left(-\frac{157809.1}{T}\right) n_{\mathrm{e}} n_{\mathrm{H}} \\
\Lambda_{\mathrm{He}}^{\text {col-ion }}=9.38 \times 10^{-22} T^{0.5}\left(1+T_{5}^{0.5}\right)^{-1} \exp \left(-\frac{285335.4}{T}\right) n_{\mathrm{e}} n_{\mathrm{He}}\end{array}$ \\
\hline$(5)$ & collisional excitation & $\begin{array}{l}\Lambda_{\mathrm{H}}^{\text {col-exc }}=7.5 \times 10^{-19}\left(1+T_{5}^{0.5}\right)^{-1} \exp \left(-\frac{118348}{T}\right) n_{\mathrm{e}} n_{\mathrm{H}} \\
\Lambda_{\mathrm{He}}^{\text {col-exc }}=9.1 \times 10^{-27} T^{-0.1687}\left(1+T_{5}^{0.5}\right)^{-1} \exp \left(-\frac{13179}{T}\right) n_{\mathrm{e}}^{2} n_{\mathrm{He}^{+}} \\
\Lambda_{\mathrm{He}^{+}}^{\text {col-exc }}=5.54 \times 10^{-17} T^{-0.397}\left(1+T_{5}^{0.5}\right)^{-1} \exp \left(-\frac{473638}{T}\right) n_{\mathrm{e}} n_{\mathrm{He}^{+}}\end{array}$ \\
\hline (6) & Bremsstrahlung & $\begin{array}{l}\Lambda^{\mathrm{Bre}}=1.42 \times 10^{-27} T^{0.5}\left(n_{\mathrm{H}^{+}}+n_{\mathrm{He}^{+}}\right) n_{\mathrm{e}} \\
\Lambda^{\mathrm{com}}=1.017 \times 10^{-37} T_{\mathrm{CMB}}^{4}\left(T-T_{\mathrm{CMB}}\right) n_{\mathrm{e}}\end{array}$ \\
\hline
\end{tabular}

${ }^{\mathrm{f}} T_{3}=T / 10^{3} \mathrm{~K}, T_{5}=T / 10^{5} \mathrm{~K}, T_{6}=T / 10^{6} \mathrm{~K}$

$\mathrm{g}$ Function (1) is from Galli \& Palla (1998) \& Hollenbach \& McKee (1979); function (2) from Glover \& Jappsen (2007); functions (3) (5) are from Maselli et al. (2003); functions (6) - (7) are from Maselli et al. (2003); original references are found in these cited papers. 